\title{
Classification of Bicovariant Differential Calculi on Quantum Groups
}

\author{
Konrad Schmüdgen, Axel Schüler \\ Fakultät für Mathematik und Informatik und Naturwiss., Theoretisches Zentrum, Universität \\ Leipzig, Augustusplatz 10, D-04109 Leipzig, Germany; E-mail schmuedgen@mathematik. \\ uni-leipzig.d400.de
}

Received: 16 May 1994/in revised form: 20 December 1994

\begin{abstract}
Suppose that $q$ is not a root of unity. We classify all bicovariant differential calculi of dimension greater than one on the quantum groups $G L_{q}(N), O_{q}(N)$ and $S p_{q}(N)$ for which the differentials $\mathrm{d} u_{j}^{i}$ of the matrix entries $u_{j}^{i}$ generate the left module of first order forms. Our first classification theorem asserts that there are precisely two one-parameter families of such calculi on $G L_{q}(N)$ for $N \geqq 3$. In the limit $q \rightarrow 1$ only two of these calculi give the ordinary differential calculus on $G L(N)$. Our second main theorem states that apart from finitely many $q$ there exist precisely two differential calculi with these properties on $O_{q}(N)$ and $S p_{q}(N)$ for $N \geqq 4$. This strengthens the corresponding result proved in our previous paper [SS2]. There are four such calculi on $O_{q}(3)$. We introduce two new 4-dimensional bicovariant differential calculi on $O_{q}(3)$.
\end{abstract}

\section{Introduction}

In recent years the theory of quantum groups $[\mathrm{D}, \mathrm{J}]$ has attracted considerable interest among both mathematicians and theoretical physicists, cf. [Mj]. Non-commutative differential calculus on quantum groups is a fundamental tool needed for many applications. For instance, it enters essentially the formulation of gauge theory with quantum groups, see e.g. [BM or C]. A general framework for bicovariant differential calculus on quantum groups has been provided by S.L. Woronowicz [W] following general ideas of A. Connes. In contrast to the classical differential geometry on Lie groups, there is no functorial method to obtain a unique bicovariant differential calculus on a given quantum group.

In this paper we classify all bicovariant differential calculi on the quantum groups $G L_{q}(N), O_{q}(N)$ and $S p_{q}(N)$ under "natural" conditions. To be precise, we assume that $q$ is not a root of unity, the differentials $\mathrm{d} u_{j}^{i}, i, j=1, \ldots, N$, generate the left module of the first order forms and that the dimension of the calculus is greater than one. Here $u=\left(u_{j}^{i}\right)_{i, j=1, \ldots, N}$ denotes the corresponding fundamental matrix.

The results of the present paper and its predecessor [SS2] provide a complete classification of all bicovariant differential calculi on the quantum groups $G L_{q}(N), S L_{q}(N), O_{q}(N)$ and $S p_{q}(N)$ under the above assumptions. They show in 
particular that for the quantum groups $S L_{q}(N), O_{q}(N)$ and $S p_{q}(N)$ there is no such bicovariant calculus whose dimension coincides with the dimension of the corresponding classical Lie group. Except for the case of $O_{q}(3)$, the dimension of all these calculi is $N^{2}$.

The first main part of the paper is concerned with the classification problem for the quantum general linear groups $G L_{q}(N)$. The results of our classification are briefly stated as follows. For $N \geqq 3$ there are two families of non-isomorphic calculi on $G L_{q}(N)$ both depending on a complex parameter. There is only a single oneparameter family of non-isomorphic calculi on $G L_{q}(2)$. The latter result is already obtained in $[\mathrm{MH}]$. All calculi occurring in this classification can be constructed by the method of B.Jurčo [Ju]. Moreover, we describe the structure of these calculi in terms of the Maurer-Cartan forms, and determine the ad-invariant right ideals of ker $\varepsilon$ associated with these calculi.

The results of the first part are in Theorems 2.1 and 2.2 of Sect. 2. The proofs of both theorems are contained in Sect. 3. The method of proof is the same as used in our previous paper [SS2] for the quantum group $S L_{q}(N)$. The crucial step to achieve the classification is the description of the structure of the associated adinvariant right ideals of ker $\varepsilon$ by decomposing parts of the adjoint representations into irreducible components. This is the point where the difference between $N=2$ and $N \geqq 3$ occurs.

The purpose of the second main part of this paper is to strengthen the classification for $O_{q}(N)$ and $S p_{q}(N)$ obtained in [SS2], Theorems 6.1 and 6.2. Recall that we assumed there that the differential $\mathrm{d} u_{j}^{i}, i, j=1, \ldots, N$, generate the left module of one forms and that the dimension of the calculus is $N^{2}$. As mentioned above, we now weaken the latter by requiring only that the dimension is greater than one. We prove that for $N \geqq 4$ the two calculi $\left(\Gamma_{ \pm}, \mathrm{d}\right)$ defined in [SS2], Sect. 6, are the only bicovariant differential calculi on $O_{q}(N)$ and $S p_{q}(N)$ satisfying the above weaker assumptions. For the quantum group $O_{p}(3)$ this is no longer true. In addition to the calculi $\left(\Gamma_{ \pm}, \mathrm{d}\right)$ there exist two other 4-dimensional bicovariant differential calculi on $O_{q}(3)$ which are introduced and studied in Sect. 4. The main results of this second part are Theorems 4.1 and 4.2. Their proofs are given in Sect. 5. As in [SS2], these proofs require a number of long computations.

With the exception of Remark 4 in Sect. 2, we assume throughout this paper that the deformation parameter $q$ is not a root of unity. We essentially use this assumption in order to have similar decompositions of (certain) tensor product representations of $u$ and $u^{c}$ as in the classical case [L,R] (see e.g. [PW]), so that these decompositions can be labelled by means of Young tableaus [Hm]. (In fact, it would be sufficient to assume that $q^{n} \neq 1$ for all $n \in \mathbb{N}$ and $n \leqq c(N)$, where $c(N)$ is some constant depending on $N$.)

Let us briefly explain the terminology and some notations used in the sequel. Throughout this paper $\mathscr{A}$ denotes the Hopf algebra of functions for one of the quantum groups $G L_{q}(N), O_{q}(N)$ or $S p_{q}(N)$ as defined in [FRT], cf. [M1]. We denote by $\Delta$ the comultiplication, $\varepsilon$ the counit, $\kappa$ the antipode and $\mathbb{1}$ the unit element of $\mathscr{A}$. Let $\hat{R}=\left(\hat{R}_{n m}^{i j}\right)$ be the corresponding $\hat{R}$-matrix and let $P_{ \pm}$, resp. $P_{0}$ be the spectral projections of $\hat{R}$. See [FRT], Sects. 1.3 and 1.4 , for details. We denote by $u=\left(u_{j}^{i}\right)$ the fundamental matrix for $\mathscr{A}$ and by $\mathscr{A}_{n}$ the linear subspace of $\mathscr{A}$ spanned by the products $u_{j_{1}}^{i_{1}} \ldots u_{j_{n}}^{i_{n}}$. The adjoint representation of $\mathscr{A}$ is the map ad : $\mathscr{A} \rightarrow \mathscr{A} \otimes \mathscr{A}$ defined by $\operatorname{ad}(a)=b_{i} \otimes \kappa\left(a_{i}\right) c_{i}$ for $a \in \mathscr{A}$, where (id $\otimes \Delta) \Delta(a)=a_{i} \otimes b_{i} \otimes c_{i}$. We put $\tilde{a}:=a-\varepsilon(x) \mathbb{1}$ for $a \in \mathscr{A}$ 
and $\tilde{\mathscr{B}}:=\{\tilde{b}: b \in \mathscr{B}\}$ for a subset $\mathscr{B}$ of $\mathscr{A}$. The (complex) linear span of $\mathscr{B} \subseteq$ $\mathscr{A}$ is denoted by $\langle\mathscr{B}\rangle$. Unless the contrary is stated, we sum over repeated indices.

Concerning bicovariant differential calculi on quantum groups we use the terminology and the results of [W]. A differential calculus over $\mathscr{A}$ is a pair $(\Gamma, \mathrm{d})$ of a bimodule $\Gamma$ for the algebra $\mathscr{A}$ and a linear mapping $d: \mathscr{A} \rightarrow \Gamma$ satisfying the Leibniz rule $\mathrm{d}(a b)=\mathrm{d} a \cdot b+a \cdot \mathrm{d} b$ for $a, b \in \mathscr{A}$ and $\Gamma=\langle a \cdot \mathrm{d} b: a, b \in \mathscr{A}\rangle$. Two differential calculi $\left(\Gamma_{1}, \mathrm{~d}_{1}\right)$ and $\left(\Gamma_{2}, \mathrm{~d}_{2}\right)$ over $\mathscr{A}$ are isomorphic if there is a bimodule isomorphism of $\Gamma_{1}$ onto $\Gamma_{2}$ which intertwines $\mathrm{d}_{1}$ and $\mathrm{d}_{2}$.

A differential calculus $(\Gamma, \mathrm{d})$ over $\mathscr{A}$ is called bicovariant if there are linear mappings $\Delta_{L}: \Gamma \rightarrow \mathscr{A} \otimes \Gamma$ and $\Delta_{R}: \Gamma \rightarrow \Gamma \otimes \mathscr{A}$ such that:

(i) $\left(\Gamma, \Delta_{L}\right)$ is a left comodule and $\left(\Gamma, \Delta_{R}\right)$ is a right comodule for $\mathscr{A}$.

(ii) $\Delta_{L}(a \omega b)=\Delta(a) \Delta_{L}(\omega) \Delta(b)$ and $\Delta_{R}(a \omega b)=\Delta(a) \Delta_{R}(\omega) \Delta(b)$ for $a, b \in \mathscr{A}$ and $\omega \in \Gamma$.

(iii) $\Delta_{L}(\mathrm{~d} a)=($ id $\otimes \mathrm{d}) \Delta(a)$ and $\Delta_{R}(\mathrm{~d} a)=(\mathrm{d} \otimes \mathrm{id}) \Delta(a)$ for $a \in \mathscr{A}$.

Let $(\Gamma, \mathrm{d})$ be a bicovariant differential calculus over $\mathscr{A}$. A form $\omega \in \Gamma$ is said to be left-invariant (resp. right-invariant) if $\Delta_{L}(\omega)=\mathbb{1} \otimes \omega\left(\right.$ resp. $\left.\Delta_{R}(\omega)=\omega \otimes \mathbb{1}\right)$. The dimension of the vector space $\Gamma_{i n v}$ of left-invariant forms is called the dimension of the calculus $(\Gamma, \mathrm{d})$. There is a one-to-one correspondence between ad-invariant right ideals $\mathscr{R}$ of ker $\varepsilon$ and bicovariant calculi $(\Gamma, \mathrm{d})$ is given by $\mathscr{R}=\left\{a \in \operatorname{ker} \varepsilon: P_{i n v}(\mathrm{~d} a)=0\right\}$. Here $P_{i n v}$ is the canonical projection of $\Gamma$ into $\Gamma_{\text {inv }}$ defined by $P_{\text {inv }}(\omega)=\kappa\left(a_{i}\right) \omega_{i}$ when $\Delta_{L}(\omega)=a_{l} \otimes \omega_{i}$. We shall write $a \equiv b$ for $a, b \in \mathscr{A}$ if $a-b \in \mathscr{R}$. Sometimes we use Sweedler's notation $\Delta(a)=a_{(1)} \otimes a_{(2)}$.

\section{Bicovariant Differential Calculi on $G L_{q}(N)$ : The Main Results}

Throughout this and the following section $\mathscr{A}$ is the Hopf algebra of functions on $G L_{q}(N)$. As an algebra, $\mathscr{A}$ is generated by the $N^{2}$ matrix entries $u_{j}^{i}$ and an element $x$ satisfying $x \mathscr{D}=\mathscr{D} x=\mathbb{1}$, where $\mathscr{D}=\sum_{\sigma}(-q)^{l(\sigma)} u_{\sigma(1)}^{1} \ldots u_{\sigma(N)}^{N}$ denotes the quantum determinant.

The ad-invariant right ideals $\mathscr{R}_{ \pm, z g}$.

First we recall some notation from [SS2]. We shall abbreviate

$$
\begin{aligned}
& \mathfrak{s}:=\sum_{i=1}^{N} q^{-2 i}, \mathfrak{s}_{+}:=1+\mathfrak{s}+q^{-2 N-2}, \mathfrak{s}_{-}:=\mathfrak{s}-q^{-2}-q^{-2 N}, \\
& \mathfrak{t}_{+}:=1+\mathfrak{s}, \mathfrak{t}_{-}:=\mathfrak{s}-q^{-2 N}, \quad \mathfrak{r}_{+}:=\mathfrak{s}+1-q^{-2}, \\
& \mathfrak{r}_{-}:=\mathfrak{s}-q^{-2 N}+q^{-2 N-2}, \quad Q:=q-q^{-1}, Q_{+}:=q+q^{-1}, \\
& \left(v_{ \pm}\right)_{j}^{i}:=\left(P_{ \pm}\right)_{n m}^{k i} q^{-2 k} u_{k}^{n} u_{j}^{m}, U:=\sum_{i=1}^{N} q^{-2 i} u_{i}^{i} \text { and } V_{ \pm}:=\sum_{i=1}^{n} q^{-2 i}\left(v_{ \pm}\right)_{l}^{i} .
\end{aligned}
$$

We define linear mappings $S_{ \pm}: \mathscr{A}_{2} \rightarrow \mathscr{A}_{2}$ by

$$
S_{ \pm}\left(u_{j}^{i} u_{m}^{n}\right):=\left(P_{ \pm} u_{1} u_{2}\right)_{j m}^{i n}-\frac{Q_{ \pm}^{2}}{\mathfrak{s}_{ \pm}}\left(P_{ \pm}\left(v_{ \pm}\right)_{2} P_{ \pm}\right)_{j m}^{i n}+\frac{1+q^{2}}{\mathfrak{s}_{ \pm} \mathfrak{t}_{ \pm}}\left(P_{ \pm}\right)_{j m}^{i n} V_{ \pm}
$$


(Lower indices refer to the usual tensor product notations. For instance, $\left(P_{ \pm} u_{1} u_{2}\right)_{j m}^{i n}$ reads as $\left(P_{ \pm}\right)_{k l}^{i n} u_{j}^{k} u_{m}^{l}$. Note that $\mathfrak{s}_{ \pm} \neq 0$ and $\mathfrak{t}_{ \pm} \neq 0$ because we assume that $q$ is not a root of unity.)

Suppose that $N \geqq 3$. We set

$$
\begin{gathered}
\lambda_{+, z}^{+}:=\mathfrak{s}_{+} Q_{+}^{-1} q z, \lambda_{+, z}^{-}:=\mathfrak{s}_{-} Q_{+}^{-1} q^{-1} z, v_{+, z}:=\left(q^{-2} z^{-N}-1\right)\left(z \mathfrak{r}_{+}-\mathfrak{s}\right)^{-1}, \\
\mu_{+, z}^{+}:=\mathfrak{t}_{+} \frac{\left(\mathfrak{s}-q^{-2}+q^{2}\right) z^{2}-\mathfrak{s}}{q Q_{+}\left(z \mathfrak{r}_{+}-\mathfrak{s}\right)}, \mu_{+, z}^{-}:=\mathfrak{t}_{+} \frac{\left(\mathfrak{s}-q^{-4}+1\right) z^{2}-\mathfrak{s}}{q Q_{+}\left(z \mathfrak{r}_{+}-\mathfrak{s}\right)},
\end{gathered}
$$

if $z \mathfrak{r}_{+} \neq \mathfrak{s}$ and

$$
\begin{aligned}
& \lambda_{-, z}^{+},:=\mathfrak{s}_{+} Q_{+}^{-1} q^{-1} z, \lambda_{-, z}^{-}:=\mathfrak{s}_{-} Q_{+}^{-1} q z, v_{-, z}:=\left(q^{2} z^{-N}-1\right)\left(z \mathfrak{x}_{-}-\mathfrak{s}\right)^{-1} \\
& \mu_{-, z}^{+}:=\mathfrak{t}_{+} \frac{\left(\mathfrak{s}-q^{-2 N}+q^{-2 N-4}\right) z^{2}-\mathfrak{s}}{q Q_{+}\left(z \mathfrak{r}_{-}-\mathfrak{s}\right)} \\
& \mu_{-, z}^{-}:=\mathbf{t}_{-} \frac{\left(\mathfrak{s}-q^{-2 N+2}+q^{-2 N-2}\right) z^{2}-\mathfrak{s}}{q Q_{+}\left(z \mathbf{r}_{-}-\mathfrak{s}\right)}
\end{aligned}
$$

if $z \mathfrak{r}_{-} \neq \mathfrak{s}$.

Let $\tau \in\{+,-\}$ and $z \in \mathbb{C} \backslash\{0\}$ be such that $z \mathfrak{r}_{\tau} \neq \mathfrak{s}$. Let $\mathscr{R}_{\tau, z}:=\mathscr{B}_{\tau, z} \cdot \mathscr{A}$ denote the right ideal of ker $\varepsilon$, where $\mathscr{B}_{\tau, z}$ is the linear subspace of ker $\varepsilon$ generated by the following three groups of elements:

(1) $S_{ \pm}\left(u_{j}^{i} u_{m}^{n}\right) ; i, j, n, m=1, \ldots, N$.

(2) $\left(v_{ \pm}\right)_{j}^{i}-\lambda_{\tau, z}^{ \pm} u_{j}^{i}-\delta_{i j} \mathfrak{s}^{-1}\left(V_{ \pm}-\lambda_{\tau, z}^{ \pm} U\right) ; i, j=1, \ldots, N$.

(3) $\tilde{V}_{+}-\mu_{\tau, k}^{+} \tilde{U}, \tilde{V}--\mu_{\tau, z}^{-} \tilde{U}, \tilde{x}-v_{\tau, z} \tilde{U}$.

Now suppose that $N=2$. Suppose that $z \in \mathbb{C} \backslash\{0\}$ and $z \mathfrak{r}_{+} \neq \mathfrak{s}$ (i.e. $z\left(q^{4}+1\right) \neq$ $\left.q^{2}+1\right)$. As above, we set $v_{+, z}:=\left(q^{-2} z^{-2}-1\right)\left(z \mathfrak{r}_{+}-\mathfrak{s}\right)^{-1}$. We define a right ideal $\mathscr{R}_{+, z}:=\mathscr{B}_{+, z} \cdot \mathscr{A}$ of ker $\varepsilon$, where $\mathscr{B}_{+, z}$ is the linear span of the following elements:

(4) $\left(u_{1}^{1}\right)^{2}+q^{2}\left(u_{2}^{2}\right)^{2}-\left(1+q^{2}\right)\left(u_{1}^{1} u_{2}^{2}+q^{-1} u_{2}^{1} u_{1}^{2}\right),\left(u_{2}^{1}\right)^{2},\left(u_{1}^{2}\right)^{2}, u_{2}^{1}\left(u_{1}^{1}-u_{2}^{2}\right)$, $u_{1}^{2}\left(u_{1}^{1}-u_{2}^{2}\right)$.

(5) $\left(U-z \mathfrak{r}_{+}\right) u_{2}^{1},\left(U-z \mathfrak{r}_{+}\right) u_{1}^{2},\left(U-z \mathfrak{r}_{+}\right)\left(u_{1}^{1}-u_{2}^{2}\right)$.

(6) $\left(U-z \mathfrak{r}_{+}\right) \tilde{U}, \tilde{x}-v_{+, z} \tilde{U}$.

The Bicovariant Differential Calculi $\left(\Gamma_{ \pm, z}, \mathrm{~d}\right)$

We define some bicovariant differential calculi by the method of B. Jurčo [Ju] adapted to the case of $G L_{q}(N)$. Suppose that $z_{+}$and $z_{-}$are non-zero complex numbers. Let $L^{ \pm}=\left({ }^{ \pm} l_{j}^{i}\right)$ be the $N \times N$ matrices of linear functionals ${ }^{ \pm} l_{j}^{l}$ on $\mathscr{A}$ as defined in Sect. 2 of [FRT] by taking the matrices $z_{ \pm} \hat{R}$ therein. Then we have

$$
\begin{aligned}
& { }^{+} l_{j}^{l}\left(u_{m}^{n}\right)=z_{+} \hat{R}_{m j}^{i n}, \quad{ }^{-} l_{j}^{i}\left(u_{m}^{n}\right)=z_{-}\left(\hat{R}^{-1}\right)_{m j}^{i n},{ }^{+} l_{j}^{i}(\mathscr{D})=z_{+}^{N} q \delta_{i j},{ }^{-} l_{j}^{i}(\mathscr{D})=z_{-}^{N} q^{-1} \delta_{i j}, \\
& \kappa\left({ }^{+} l_{j}^{i}\right)\left(u_{m}^{n}\right)=z_{+}^{-1}\left(\hat{R}^{-1}\right)_{j m}^{n i} \text { and } \kappa\left(-l_{j}^{i}\right)\left(u_{m}^{n}\right)=z_{-}^{-1} \hat{R}_{j m}^{n i} .
\end{aligned}
$$


(In order to verify the formulas for the quantum determinant $\mathscr{D}$, it suffices to note that $\pm l_{j}^{i}(\mathscr{D})= \pm l_{j}^{i}(\mathbb{1})$ if $z_{ \pm}^{N}=q^{\mp 1}$, cf. [FRT], 2.2.)

Similarly as in case of quantized simple Lie groups (see [Ju] or [SS2] for details),

$$
\begin{aligned}
& \Gamma_{+, z}:=\left(u^{c}, L^{+}\right) \otimes\left(u, L^{-, c}\right) \text { for } z=z_{+} z_{-}^{-1} \text { and } \\
& \Gamma_{-, z}:=\left(u^{c}, L^{-}\right) \otimes\left(u, L^{+, c}\right) \text { for } z=z_{-} z_{+}^{-1}
\end{aligned}
$$

define bicovariant bimodules over $\mathscr{A}=G L_{q}(N)$. Here $u^{c}$ and $L^{ \pm, c}$ denote the contragredient representations of $u$ and $L^{ \pm}$, respectively. The structure of $\Gamma_{ \pm, z}$ is easily described as follows: There exists a basis $\left\{\eta_{i j}: i, j=1, \ldots, N\right\}$ of the vector space $\left(\Gamma_{ \pm, z}\right)_{i n v}$ of left invariant elements of $\Gamma_{ \pm, z}$ such that the bimodule operations of $\Gamma_{ \pm, z}$ and the right action $\Delta_{R}$ on $\left(\Gamma_{ \pm, z}\right)_{i n v}$ are given by

$$
\eta_{i j} a=\left({ }^{ \pm} l_{n}^{i} \kappa\left({ }^{\mp} l_{j}^{m}\right) * a\right) \eta_{n m}, a \in \mathscr{A},
$$

and $\Delta_{R}\left(\eta_{i j}\right)=\eta_{n m} \otimes\left(u^{c}\right)_{i}^{n} u_{j}^{m}, i, j=1, \ldots, N$

Since $\left(u^{c}\right)_{i}^{n} u_{i}^{m} q^{-2 i}=\kappa\left(u_{n}^{i}\right) u_{i}^{m} q^{-2 i}=\delta_{n m} q^{-2 n}$, the element $\eta:=\sum_{i} q^{-2 i} \eta_{i i}$ of $\Gamma_{ \pm, z}$ is left-and right-invariant. Defining $\mathrm{d} a:=\eta a-a \eta$ for $a \in \mathscr{A}$, the pair $\left(\Gamma_{ \pm, z}, \mathrm{~d}\right)$ becomes a bicovariant differential calculus over $\mathscr{A}$.

Further, we set $K_{n m}^{i j}:=q^{-2 i} \delta_{i j} \delta_{n m}, \hat{R}_{n m}^{i j}:=\hat{R}_{m j}^{n i}$ and $\left(\hat{R}^{-}\right)_{n m}^{i j}:=\left(\hat{R}^{-1}\right)_{m j}^{n i}$. For $z \mathfrak{r}_{ \pm} \neq \mathfrak{s}$, we define linear mappings $T_{ \pm, z} \in L\left(\mathbb{C}^{N} \otimes \mathbb{C}^{N} \otimes \mathbb{C}^{N}\right)$ by

$$
T_{+, z}:=z\left(X_{+, z}\right)_{23}^{-1} \dot{R}_{12} \hat{R}_{23}\left(X_{+, z}\right)_{12} \text { and } T_{-, z}:=z\left(X_{-, z}\right)_{23}^{-1} \dot{R}_{12}^{-} \hat{R}_{23}^{-1}\left(X_{-, z}\right)_{12}
$$

where $X_{+, z}:=I+\left(1-z^{-1}\right) q Q^{-1} K$ and $X_{-, z}:=I+\left(z^{-1}-1\right) q^{2 N+1} Q^{-1} K$.

(Since $z \mathfrak{r}_{ \pm} \neq \mathfrak{s}$ by assumption, it is easy to check that $X_{ \pm, z}$ is invertible in $L\left(\mathbb{C}^{n} \otimes\right.$ $\left.\mathbb{C}^{N}\right)$ and that $\left.X_{ \pm, z}^{-1}=I+\left(1-z^{-1}\right)\left(z \mathfrak{r}_{ \pm}-\mathfrak{s}\right)^{-1} K.\right)$

The main results in the first half of this paper are summarized in the following two theorems.

Theorem 2.1. Suppose that $\mathscr{A}=G L_{q}(N)$ with $N \geqq 3$.

(i) The right ideals $\mathscr{R}_{ \pm, z}$ for $z \mathfrak{r}_{ \pm} \neq \mathfrak{s}$ are the only ad-invariant right ideals $\mathscr{R}$ of ker $\varepsilon$ such that $\operatorname{ker} \varepsilon=\mathscr{R}+\tilde{\mathscr{A}_{1}}$ and codim $\mathscr{R} \geqq 2$. Moreover, codim $\mathscr{R}_{ \pm, z}=N^{2}$ and $\mathscr{R}_{\tau, z} \neq \mathscr{R}_{\tau^{\prime}, z^{\prime}}$ if $(\tau, z) \neq\left(\tau^{\prime}, z^{\prime}\right)$.

(ii) The pairs $\left(\Gamma_{ \pm, z}, \mathrm{~d}\right)$ with $z \mathfrak{r}_{ \pm} \neq \mathfrak{s}$ are the only bicovariant differential calculi $(\Gamma, \mathrm{d})$ over $\mathscr{A}=G L_{q}(N)$ for which $\operatorname{dim} \Gamma_{\text {inv }} \geqq 2$ and $\Gamma=\left\langle a \cdot \mathrm{d} u_{j}^{i}: a \in \mathscr{A}, i, j=\right.$ $1, \ldots, N\rangle$. Two calculi $\left(\Gamma_{\tau, z}, \mathrm{~d}\right)$ and $\left(\Gamma_{\tau^{\prime}, z^{\prime}}, \mathrm{d}\right)$ with $(\tau, z) \neq\left(\tau^{\prime}, z^{\prime}\right)$ are non-isomorphic. The pair $\left(\Gamma_{ \pm, z}, \mathrm{~d}\right)$ is the canonical calculus associated with the ad-invariant right ideal $\mathscr{R}_{ \pm, z}$.

(iii) Suppose that $z \mathfrak{r}_{ \pm} \neq \mathfrak{s}$. The set of one forms $\omega_{l j}:=\kappa\left(u_{n}^{i}\right) \mathrm{d} u_{j}^{n}, i, j=1, \ldots, N$, is a vector space basis of $\left(\Gamma_{ \pm, z}\right)_{i n v}$. The bimodule structure and the differentiation of the calculus $\left(\Gamma_{ \pm, z}, \mathrm{~d}\right)$ can be rewritten as

$$
\omega_{i j} a=\left(f_{n m}^{i j} * a\right) \omega_{n m} \text { and } \mathrm{d} a=\left(\chi_{n m} * a\right) \omega_{n m}, a \in \mathscr{A},
$$


where the linear functionals $f_{n m}^{i j}$ and $\chi_{n m}$ on $\mathscr{A}$ satisfy

$$
\chi_{n m}\left(u_{j}^{i}\right)=\delta_{i n} \delta_{j m} \text { and } \chi_{n m}\left(\tilde{u}_{j}^{i} u_{s}^{r}\right)=f_{n m}^{i j}\left(u_{s}^{r}\right)=\left(T_{ \pm, z}\right)_{i j s}^{r n m} .
$$

If $\omega$ denotes the left-and right-invariant one form $\sum q^{-2 i} \omega_{i i}$, then we have

$$
\begin{aligned}
\mathrm{d} a & =\left(z \mathfrak{r}_{ \pm}-\mathfrak{s}\right)^{-1}(\omega a-a \omega) \text { for } a \in \mathscr{A} \\
\text { and } \quad \mathrm{d} \mathscr{D} & =-z^{N} q^{ \pm 2} v_{ \pm, z} \mathscr{D} \omega, \mathrm{d} x=v_{ \pm, z} x \omega \text { and } \mathrm{d} x=-z^{-N} q^{\mp 2} x^{2} \mathrm{~d} \mathscr{D}
\end{aligned}
$$

Theorem 2.2. Suppose that $\mathscr{A}=G L_{q}(2)$. Then the pairs $\left(\Gamma_{+, z}, \mathrm{~d}\right)$ with $z\left(q^{4}+1\right) \neq$ $q^{2}+1$ are the only bicovariant differential calculi $(\Gamma, \mathrm{d})$ over $\mathscr{A}$ such that $\operatorname{dim}(\Gamma, \mathrm{d}) \geqq 2$ and $\Gamma=\left\langle a \mathrm{~d} u_{j}^{i}: a \in \mathscr{A}, i, j=1,2\right\rangle$. All assertions of Theorem 2.1 concerning the calculus $\left(\Gamma_{+, z}, \mathrm{~d}\right)$ and the right ideal $\mathscr{R}_{+, z}$ remain valid for $N=2$.

The proofs of both theorems will be given in Sect. 3. Here we discuss these results and the bicovariant differential calculi occuring therein by some remarks.

1) For $z \mathfrak{r}_{ \pm}=\mathfrak{s}$ the pair $\left(\Gamma_{ \pm, z}, \mathrm{~d}\right)$ is still a well-defined bicovariant differential calculus over $\mathscr{A}$, but the differentials $\mathrm{d} u, i, j=1, \ldots, N$, do not generate the left module $\Gamma_{ \pm, z}$ in this case.

2) In [SS2] all bicovariant differential calculi on $S L_{q}(N)$ have been classified under the assumptions stated in the introduction. A direct comparison with [SS2] shows that all calculi of this list can be obtained from the calculi $\left(\Gamma_{ \pm, z}, \mathrm{~d}\right)$ on $G L_{q}(N)$ for special values of the parameter $z$, i.e. by setting $z^{N} q^{2}=1$ for $\left(\Gamma_{+, z}, \mathrm{~d}\right)$ and $z^{n} q^{-2}=1$ for $\left(\Gamma_{-, z}, \mathrm{~d}\right)$. That for the latter values of $z$ the calculi $\left(\Gamma_{ \pm z,}, \mathrm{~d}\right)$ on $G L_{q}(N)$ factorize to the quotient algebra $S L_{q}(N)$ follows also at once from the above formulas, since then ${ }^{ \pm} l_{j}^{i}(\mathscr{D})=^{ \pm} l_{j}^{i}(\mathbb{1})$ and $\mathrm{d}(\mathscr{D})=\mathrm{d} x=0$.

3) For the calculi $\left(\Gamma_{ \pm, z}, \mathrm{~d}\right)$ with $z=1$ the commutation relations between the matrix entries and their differentials are even linear. Indeed, for $z=1$ we have $X_{ \pm, z}=I$ and (2.1) implies that $\mathrm{d} u_{j}^{i} u_{s}^{r}=\left(\hat{R}^{ \pm 1}\right)_{k l}^{i r}\left(\hat{R}^{ \pm 1}\right)_{j s}^{n m} u_{n}^{k} \mathrm{~d} u_{m}^{l}$ or in matrix notation

$$
\mathrm{d} u_{1} \cdot u_{2}=\hat{R}_{12}^{ \pm 1} u_{1} \cdot \mathrm{d} u_{2} \hat{R}_{12}^{ \pm 1}
$$

The particular bicovariant calculi $\left(\Gamma_{ \pm, 1}, \mathrm{~d}\right)$ on $G L_{q}(N)$ have been found in [M2 and M1] and studied in [S and SWZ]. Moreover, it follows easily from (2.1) that $z=1$ is the only parameter value for which $\left(\Gamma_{+, z}, \mathrm{~d}\right)$ or $\left(\Gamma_{-, z}, \mathrm{~d}\right)$ has linear commutation rules between the matrix elements and their differentials. Recall from the preceding remark that for $z=1$ the calculi $\left(\Gamma_{ \pm, z}, \mathrm{~d}\right)$ do not factorize to the quantum group $S L_{q}(N)$.

4) What about the classical limit $q \rightarrow 1$ of the calculi $\left(\Gamma_{ \pm, z}, \mathrm{~d}\right)$ ?

First we have to explain how this limit should be defined. We will adopt the viewpoint of [SS2]. (Note that our definition of the classical limit differs from the one used at various places of the literature, see e.g. [CSSW].) As in [SS2], we keep the Maurer-Cartan basis $\left\{\omega_{i j}\right\}$ of the calculus $\left(\Gamma_{ \pm, z}, \mathrm{~d}\right)$ fixed and we take the limit of the linear transformation $T_{ \pm, z}$. After some calculations it follows that for each fixed (!) number $z \in \mathbb{C} \backslash\{0\}$ the limits of $T_{+, z}$ and of $T_{-, z}$ for $q \rightarrow 1$ are

$$
T_{z}:=z \dot{R}_{12} \hat{R}_{23}+(z-1) \dot{I}_{12}-(z-1) N^{-1} K_{23} \dot{I}_{12}
$$


where $i_{n m}^{i j}=\delta_{n m} \delta_{i j}$. Replacing $T_{ \pm, z}$ by $T_{z}$, the formulas (2.1) and (2.2) in Theorem 2.1 define a bicovariant differential calculus $\left(\Gamma_{z}, \mathrm{~d}\right)$ over $\mathscr{A}=G L_{q}(N)$ for $q=1$. We consider $\left(\Gamma_{z}, \mathrm{~d}\right)$ as the classical limit of $\left(\Gamma_{ \pm, z}, \mathrm{~d}\right)$.

Let $\mathscr{R}_{z}$ denote the right ideal of ker $\varepsilon$ which is defined in the same way as $\mathscr{R}_{ \pm, z}$ with all coefficients occurring in (1)-(6) replaced with their limits for $q \rightarrow 1$ and for fixed $z$. It is not difficult to check $\mathscr{R}_{z}$ is the ad-invariant right ideal associated with the bicovariant calculus $\left(\Gamma_{z}, \mathrm{~d}\right)$. (Of course, one may also define other possible classical limits of the linear transformations $T_{ \pm, z}$ by allowing that $z$ varies as $q \rightarrow$ 1. For instance, this has been implicitly done in [SS2] for the quantum group $S L_{q}(N)$.)

If $z=1$, then $\left(T_{z}\right)_{i j s}^{r n m}=\delta_{i n} \delta_{j m} \delta_{r s}$ by (2.3). Hence we have $\omega_{i j} u_{s}^{r}=u_{s}^{r} \omega_{i j}$ for $i, j, r, s=1, \ldots, N$. From this it follows immediately that $\left(\Gamma_{1}, \mathrm{~d}\right)$ is just the classical bicovariant differential calculus on the group $G L(N)$. (Another way to prove this assertion is to verify that $\mathscr{R}_{1}$ coincides with the right ideal $(\operatorname{ker} \varepsilon)^{2}$ of the classical calculus.) Note that the Maurer-Cartan forms $\omega_{i j}$ and the matrix entries $u_{s}^{r}$ do not commute in general for the calculus $\left(\Gamma_{z}, \mathrm{~d}\right)$ if $z \neq 1$.

\section{Proofs of Theorems 2.1 and 2.2}

The proof follows closely the lines of the proofs of Theorems 2.2 and 2.3 in [SS2] where the corresponding problem for the quantum groups $S L_{q}(N)$ was treated. Suppose $\mathscr{R}$ is an ad-invariant right ideal of ker $\varepsilon$ such that ker $\varepsilon=\mathscr{R}+\tilde{\mathscr{A}_{1}}$ and $\operatorname{codim} \mathscr{R} \geqq 2$. Let $\mathscr{R}_{12}:=\mathscr{R} \cap\left(\mathscr{A}_{1}+\mathscr{A}_{2}+\mathbb{C} \cdot x\right)^{\sim}$. Since ker $\varepsilon=\mathscr{R}+\tilde{\mathscr{A}}_{1}$ by assumption, a simple induction argument (cf. Lemma 1.1 in [SS2]) proves that $\mathscr{R}=\mathscr{R}_{12} \cdot \mathscr{A}$.

Assume first that $N \geqq 3$. Our aim is to show that $\mathscr{R}=\mathscr{R}_{\tau, z}$ for some $\tau \in\{+,-\}$ and for some complex number $z$ such that $z \neq 0$ and $z \mathfrak{r}_{\tau} \neq \mathfrak{s}$. This is in fact the main part of the proof of Theorem 2.1.

The following lemma corresponds to Lemma 4.3 in [SS2].

Lemma 3.1. There are numbers $\delta \in\{0,1\}$ and $\lambda^{+}, \lambda^{-}, \mu^{+}, \mu^{-}, v \in \mathbb{C}$ such that

$$
\begin{aligned}
\mathscr{R}_{12}= & \delta\langle\tilde{U}\rangle \oplus\left\langle\tilde{V}_{+}-\mu^{+} \tilde{U}\right\rangle \oplus\left\langle\tilde{V}_{-}-\mu^{-} \tilde{U}\right\rangle \oplus\langle\tilde{x}-v \tilde{U}\rangle \oplus \\
& \mathscr{W}_{+} \oplus \mathscr{W} \mathscr{W}_{-} \oplus \quad \operatorname{im} S_{+} \oplus \operatorname{im} S_{-},
\end{aligned}
$$

where $\mathscr{W}_{ \pm}$is the linear span of $\left(v_{ \pm}\right)_{j}^{i}-\lambda^{ \pm} u_{j}^{i}-\delta_{i j} \mathfrak{s}^{-1}\left(V_{ \pm}-\lambda^{ \pm} U\right), i, j=1, \ldots, N$.

Proof. First suppose that $N \geqq 4$. Since obviously $\operatorname{ad}(x)=x \otimes \mathbb{1}$, the restriction to $\mathscr{R}_{12}$ of the adjoint representation decomposes as a direct sum of irreducible components as follows:

$$
\operatorname{ad}\left[\mathscr{R}_{12} \cong(3+\delta)[0] \oplus 2\left[2,1^{N-2}\right] \oplus\left[4,2^{N-2}\right] \oplus\left[2,2,1^{N-4}\right]\right.
$$

where $\delta=0$ or $\delta=1$. (We use the standard notation for the Young patterns, see e.g. $[\mathrm{Hm}]$.) The corresponding representation spaces in (3.2) are the first four direct summands of $(3.1)$ for $(3+\delta)[0], \mathscr{W}_{+}$and $\mathscr{W}_{-}$for $2\left[2,1^{N-2}\right]$, im $S_{+}$for $\left[4,2^{N-2}\right]$ and $\mathrm{im} S_{-}$for $\left[2,2,1^{N-4}\right]$. The proofs of these facts are quite similar to the proofs of the corresponding assertions for $S L_{q}(N)$ in [SS2]. We omit the details and refer to $[\mathrm{SS} 2]$. 
If $N=3$, then the representation for the Young pattern [2,2, $\left.1^{N-4}\right]$ does not occur in (3.2) and $S_{-} \equiv 0$, so the assertion is also true for $N=3$.

Lemma 3.2. Setting $\Lambda_{ \pm}:=\mathfrak{s}_{ \pm}^{-1} \lambda^{ \pm}$and $M_{ \pm}:=q\left(\mathrm{t}_{ \pm} \mathfrak{s}_{ \pm}\right)^{-1} \mu^{ \pm}$, we have the following four equations:

(i) $1=Q_{+}^{2} \Lambda_{+}^{2}\left(\Lambda_{+}+\Lambda_{-}\right)^{N-2}\left(1+v\left(\mathfrak{s}_{+} \Lambda_{+}+\mathfrak{s}_{-} \Lambda_{-}-\mathfrak{s}\right)\right)$.

(ii) $0=\left(q^{-1} \Lambda_{+}-q \Lambda_{-}\right)\left(q \Lambda_{+}-q^{-1} \Lambda_{-}\right)$.

(iii) $\left(\Lambda_{+}+\Lambda_{-}\right)^{2}=Q_{+}^{2} \Lambda_{+}\left(\Lambda_{+}+\Lambda_{-}-1\right)+Q_{+} \mathfrak{s}^{-1}\left(\mathfrak{s}_{+} M_{+}-Q_{+} \Lambda_{+}\right)$ $\times\left(\mathfrak{s}_{+} \Lambda_{+}+\mathfrak{s}_{-} \Lambda_{-}-\mathfrak{s}\right)+1$.

(iv) $0=\left(q^{-1} \Lambda_{+}-q \Lambda_{-}\right)\left(q Q_{+} \Lambda_{+}-1\right)+\left(q \Lambda_{+}-q^{-1} \Lambda_{-}\right)\left(q^{-1} Q_{+} \Lambda_{+}-1\right)$ $+_{\mathfrak{s}}^{-1}\left(\mathfrak{s}_{+} M_{+}-\mathfrak{s}_{-} M_{-}-Q_{+}\left(\Lambda_{+}-\Lambda_{-}\right)\right)\left(\mathfrak{s}_{+} \Lambda_{+}+\mathfrak{s}_{-} \Lambda_{-}-\mathfrak{s}\right)$.

Proof. The equations (ii)-(iv) appeared already in Lemma 4.5 of [SS2]. Their proof for $G L_{q}(N)$ is verbatim the same as for $S L_{q}(N)$. We verify (i). The proof of Lemma 4.5, (i), of [SS2] shows that $u_{2}^{1} \mathscr{D}-Q_{+}^{2} \Lambda_{+}^{2}\left(\Lambda_{+}+\Lambda_{-}\right)^{N-2} u_{2}^{1} \in \mathscr{R}$ in the present situation for $G L_{q}(N)$. Put $\alpha:=Q_{+}^{2} \Lambda_{+}^{2}\left(\Lambda_{+}+\Lambda_{-}\right)^{N-2}$. Since $\tilde{x} \equiv v \tilde{U}$ and $\tilde{U} u_{2}^{1}=\left(v_{+}\right)_{2}^{1}+\left(v_{-}\right)_{2}^{1}-\mathfrak{s} u_{2}^{1} \equiv\left(\lambda^{+}+\lambda^{-}-\mathfrak{s}\right) u_{2}^{1}$ by Lemma 3.1, it follows that

$$
\begin{aligned}
u_{2}^{1}-\alpha x u_{2}^{1}=u_{2}^{1}-\alpha \tilde{x} u_{2}^{1}-\alpha u_{2}^{1} & \equiv(1-\alpha) u_{2}^{1}-\alpha \nu \tilde{U} u_{2}^{1} \\
& \equiv\left(1-\alpha-\alpha \nu\left(\lambda^{+}+\lambda^{-}-\mathfrak{s}\right)\right) u_{2}^{1} \equiv 0
\end{aligned}
$$

Similarly as in [SS2], Lemma 4.2 , we have $u_{2}^{1} \notin \mathscr{R}$, since otherwise codim $\mathscr{R}=1$. Therefore, the preceding implies that $1-\alpha-\alpha v\left(\lambda^{+}+\lambda^{-}-\mathfrak{s}\right)=0$ which in turn gives (i).

Lemma 3.3. $\lambda^{+}+\lambda^{-}-\mathfrak{s} \equiv \mathfrak{s}_{+} \Lambda_{+}+\mathfrak{s}_{-} \Lambda_{-}-\mathfrak{s} \neq 0$.

Proof. Assume the contrary, that is, $\lambda^{+}+\lambda^{-}-\mathfrak{s}=0$. By Lemma 3.2, (ii), we have $\left(q^{-1} \Lambda_{+}-q \Lambda_{-}\right)\left(q \Lambda_{+}-q^{-1} \Lambda_{-}\right)=0$. First we consider the case $q^{-1} \Lambda_{+}=q \Lambda_{-}$. Then, by (iv), $\left(q \Lambda_{+}-q^{-1} \Lambda_{-}\right)\left(q^{-1} Q_{+} \Lambda_{+}-1\right)=0$. Since $q^{4} \neq 1, q \Lambda_{+}-q^{-1} \Lambda_{-}=0$ leads to $\Lambda_{+}=\Lambda_{-}=0$ which contradicts (i). Thus $\Lambda_{+}=q Q_{+}^{-1}$ and hence $\Lambda_{+}+$ $\Lambda_{-}=1$ which also contradicts (i). The second possible case $q \Lambda_{+}=q^{-1} \Lambda_{-}$by (ii) is treated in a similar manner.

Next we show that the number $\delta$ occurring in Lemma 3.1 is zero. Assume to the contrary that $\delta=1$. Then $\tilde{U} \in \mathscr{R}$ and hence $\tilde{U} u_{2}^{1}=\left(\lambda^{+}+\lambda^{-}-\mathfrak{s}\right) u_{2}^{1} \in \mathscr{R}$. Since $u_{2}^{1} \notin \mathscr{R}$ as noted above, we conclude that $\lambda^{+}+\lambda^{-}-\mathfrak{s}=0$. This is impossible by Lemma 3.3, hence we have $\delta=0$.

From Lemma 3.2, (ii), $q^{-1} \Lambda_{+}=q \Lambda_{-}$or $q \Lambda_{+}=q^{-1} \Lambda_{-}$. Let us begin with the case $q^{-1} \Lambda_{+}=q \Lambda_{-}$. Set $z:=\lambda^{+} \mathfrak{s}_{+}^{-1} Q_{+} q^{-1}$. Then we get $\lambda^{+}=\mathfrak{s}_{+} Q_{+}^{-1} q z=\lambda_{+, z}^{+}$ and $\lambda^{-}=\mathfrak{s}_{-} Q_{+}^{-1} q^{-1} z=\lambda_{+, z}^{-}$. Note that $z \neq 0$ (otherwise $\Lambda_{+}=\Lambda_{-}=0$ which contradicts Lemma 3.3) and $z \mathfrak{r}_{+} \neq \mathfrak{s}$ (otherwise $z \mathfrak{r}_{+}-\mathfrak{s}=\lambda^{+}+\lambda^{-}-\mathfrak{s}=0$ which contradicts Lemma 3.3). From the equations (i), (iii) and (iv) of Lemma 3.2 we compute easily that $\mu^{+}=\mu_{+, z}^{+}, \mu^{-}=\mu_{+, z}^{-}$and $v=v_{+, z}$. By Lemma 3.1, we have shown that $\mathscr{R}_{12}=\mathscr{B}_{+, z}$. Since $\mathscr{R}=\mathscr{R}_{12} \cdot \mathscr{A}$ as noted above and $\mathscr{R}_{+, z}=\mathscr{B}_{+, z} \cdot \mathscr{A}$ by definition, $\mathscr{R}=\mathscr{R}_{+, z}$. Arguing in a similar way, it follows that $\mathscr{R}=\mathscr{R}_{-, z}$ in the second possible case $q \Lambda_{+}=q^{-1} \Lambda_{-}$. 
Suppose now that $N=2$. We will show that $\mathscr{R}=\mathscr{R}_{+, z}$ for some $z \in \mathbb{C} \backslash\{0\}$ such that $z\left(q^{4}+1\right) \neq q^{2}+1$.

Lemma 3.4. There exist numbers $\delta \in\{0,1\}$ and $\lambda, \mu, v \in \mathbb{C}$ such that

$$
\mathscr{R}_{12}=\delta\langle\tilde{U}\rangle \oplus\langle\tilde{x}-v \tilde{U}\rangle \oplus\langle(U-\mu) \tilde{U}\rangle \oplus \mathscr{W} \oplus \mathscr{V},
$$

where $\mathscr{W}$ is the linear span of $(U-\lambda) u_{2}^{1},(U-\lambda) u_{1}^{2},(U-\lambda)\left(u_{1}^{1}-u_{2}^{2}\right)$ and $\mathscr{V}$ is the linear span of the five elements of group (4).

Proof. First we decompose $\operatorname{ad}\left[\mathscr{A}_{2}\right.$ into irreducible representations. Since $\operatorname{ad}\left(u_{j_{1}}^{i_{1}} u_{j_{2}}^{i_{2}}\right)$ $=u_{m_{1}}^{n_{1}} u_{m_{2}}^{n_{2}} \otimes\left(u^{c}\right)_{t_{2}}^{n_{2}}\left(u^{c}\right)_{i_{1}}^{n_{1}} u_{j_{1}}^{m_{1}} u_{j_{2}}^{m_{2}}$ by definition and $u^{c} \cong u$ by $N=2$, we have

$$
\operatorname{ad}\left[\mathscr{A}_{2} \subseteq\left(u^{c} \otimes u^{c}\right) \otimes(u \otimes u) \cong([0] \oplus[2]) \otimes([0] \oplus[2]) .\right.
$$

Obviously, the two projections for the decomposition $u \otimes u \cong[0] \oplus[2]$ are the spectral projections $P_{+}$and $P_{-}$of $\hat{R}$. Since $\left(P_{ \pm}\right)_{m n}^{i j}\left(P_{\mp}\right)_{r s}^{k l} u_{k}^{m} u_{l}^{n}=u_{m}^{i} u_{n}^{j}\left(P_{ \pm}\right)_{k l}^{m n}\left(P_{\mp}\right)_{r s}^{k l}=0$, we conclude that ad $\left[\operatorname{im} P_{+} \otimes P_{-}=\operatorname{ad}\left[\operatorname{im} P_{-} \otimes P_{+}=0\right.\right.$ for the decomposition at the right-hand side of (3.3). Clearly, [2] $\otimes[2] \cong[0] \oplus[2] \oplus[4]$. Comparing the corresponding dimensions, it follows that $\operatorname{ad}\left[\mathscr{A}_{2} \cong 2[0] \oplus[2] \oplus[4]\right.$.

Combined with $\operatorname{ad}\left[\mathscr{A}_{1} \cong u^{c} \otimes u \cong[0] \oplus[2]\right.$ and $\operatorname{ad}(x)=x \otimes \mathbb{1}$, we obtain $\operatorname{ad}\left[\left(\mathscr{A} \oplus \mathscr{A}_{2} \oplus \mathbb{C} \cdot x\right) \cong 4[0] \oplus 2[2] \oplus[4]\right.$ and hence $\operatorname{ad}\left[\left(\mathscr{A}_{1} \oplus \mathscr{A}_{2} \oplus \mathbb{C} \cdot x\right)^{\sim} \cong 3\right.$ $[0] \oplus 2[2] \oplus$ [4]. Recall that $\mathscr{R}_{12}+\tilde{\mathscr{A}_{1}}=\left(\mathscr{A}_{1} \oplus \mathscr{A}_{2} \oplus \mathbb{C} \cdot x\right)^{\sim}$ by the assumption $\mathscr{R}+\tilde{\mathscr{A}_{1}}=$ ker $\varepsilon$. Obviously, the representation space for the Young pattern [2] in the decomposition of ad[$\tilde{\mathscr{A}}_{1}$ is $\left(I-P_{0}\right) \tilde{\mathscr{A}}_{1}$, where $P_{0}:=\mathfrak{s}^{-1} K$. We have $(I-$ $\left.P_{0}\right) \tilde{\mathscr{A}}_{1} \cap \mathscr{R}_{12}=\{0\}$, since otherwise $\left(I-P_{0}\right) \tilde{\mathscr{A}}_{1} \subseteq \mathscr{R}_{12}$ and hence codim $\mathscr{R} \leqq 1$. Putting the preceding together, we conclude that

$$
\operatorname{ad}\left\lceil\mathscr{R}_{12} \cong(2+\delta)[0] \oplus[2] \oplus[4]\right.
$$

with $\delta \in\{0,1\}$. The space for the Young pattern [4] in (3.4) is the five dimensional vector space $\mathscr{V}$. By construction, the space $\mathscr{W}$ corresponding to the Young pattern [2] in (3.4) is an ad-invariant subspace of $\left(I-P_{0}\right) \tilde{\mathscr{A}}_{1} \oplus \tilde{U} \cdot\left(I-P_{0}\right) \tilde{\mathscr{A}}_{1}$. (The dot denotes the multiplication in the algebra $\mathscr{A}$.) Since $\left(I-P_{0}\right) \mathscr{A}_{1} \cap \mathscr{R}_{12}=\{0\}$ as already noted above, the space $\mathscr{W}$ is of the form $(\tilde{U}-\lambda \mathbb{1}) \cdot\left(I-P_{0}\right) \tilde{\mathscr{A}}_{1}$. The subspace for the trivial representations $(2+\delta)[0]$ in $(3.4)$ is contained in $\langle\tilde{U}, \tilde{U} \cdot U, \tilde{x}\rangle$, hence it can be written in the form stated above.

Lemma 3.5 Setting $z:=\mathfrak{r}_{+}^{-1} \lambda=\left(1+q^{-4}\right)^{-1} \lambda$, we have $u_{2}^{1}\left(\mathscr{D}-q^{2} z^{2}\right) \in \mathscr{R}$ and

$$
q^{2} z^{2}\left(1+v\left(z \mathfrak{r}_{+}-\mathfrak{s}\right)\right)=1 \text {. }
$$

Proof. Recall that $u_{2}^{1}\left(u_{1}^{1}-u_{2}^{2}\right),\left(u_{2}^{1}\right)^{2} \in \tilde{\mathscr{V}} \subseteq \mathscr{R}_{12}$ and $\left(U-\left(1+q^{-4}\right) z\right) u_{2}^{1} \in \mathscr{R}$ by Lemma 3.4. Therefore, we have $u_{1}^{1} u_{2}^{1}=q u_{2}^{1} u_{1}^{1} \equiv q u_{2}^{1} u_{2}^{2}=q^{2} u_{2}^{2} u_{2}^{1}$ and $(U-(1+$ $\left.\left.q^{-4}\right) z\right) u_{2}^{1}=\left(1+q^{-4}\right)\left(u_{2}^{2} u_{2}^{1}-z u_{2}^{1}\right) \in \mathscr{R}$. Since $1+q^{-4} \neq 0, u_{2}^{2} u_{2}^{1} \equiv z u_{2}^{1}$, and hence $u_{2}^{1} \mathscr{D}=\mathscr{D} u_{2}^{1}=\left(u_{1}^{1} u_{2}^{2}-q u_{2}^{1} u_{1}^{2}\right) u_{2}^{1} \equiv z u_{1}^{1} u_{2}^{1}=z q u_{2}^{1} u_{1}^{1} \equiv z q u_{2}^{1} u_{2}^{2}=z q^{2} u_{2}^{2} u_{2}^{1} \equiv z^{2} q^{2} u_{2}^{1}$, which proves the first assertion. From $u_{2}^{1}\left(\mathscr{D}-q^{2} z^{2}\right) \equiv 0$ we obtain $0 \equiv u_{2}^{1}(1-$ $\left.q^{2} z^{2} x\right)=u_{2}^{1}\left(1-q^{2} z^{2}\right)-q^{2} z^{2} \tilde{x} u_{2}^{1} \equiv u_{2}^{1}\left(1-q^{2} z^{2}\right)-q^{2} z^{2} v \tilde{U} u_{2}^{1} \equiv u_{2}^{1}\left(1-q^{2} z^{2}\right)-q^{2} z^{2} v$ $\left(z \mathfrak{r}_{+}-\mathfrak{s}\right) u_{2}^{1}$. Since $u_{2}^{1} \notin \mathscr{R}$ as noted above, (3.5) follows. 
Next we prove that $\delta=0$. Assume to the contrary that $\delta=1$. Then $\tilde{U} \in \mathscr{R}$, so $(U-\mathfrak{s}) u_{2}^{1} \in \mathscr{R}$ and $\left(U-z \mathfrak{r}_{+}\right) u_{2}^{1} \in \mathscr{R}$. From $u_{2}^{1} \notin \mathscr{R}$, we get $z\left(1+q^{-4}\right)=z \mathfrak{r}_{+}=$ $\mathfrak{s}=q^{-2}+q^{-4}$, and hence $q^{2} z^{2}=1$ by (3.5). The two latter equations imply that $q$ is a root of unity which contradicts our standing assumption. Thus $\delta=0$. The latter argument also shows that $\lambda=z \mathfrak{r}_{+} \neq \mathfrak{s}$. Moreover, we have $z \neq 0$, since otherwise $u_{2}^{1} \mathscr{D} \in \mathscr{R}$, and hence $u_{2}^{1}=u_{2}^{1} \mathscr{D} \tilde{x}+u_{2}^{1} \mathscr{D} \in \mathscr{R}$. From (3.5) we compute that $v=\left(q^{-2} z^{-2}-1\right)\left(z \mathfrak{r}_{+}-\mathfrak{s}\right)^{-1}$. Further, from $(U-\lambda) u_{2}^{1} \in \mathscr{R}$ and $(U-\mu) \tilde{U} u_{2}^{1} \in$ $\mathscr{R}$, we conclude that $(\lambda-\mu)(\lambda-\mathfrak{s}) u_{2}^{1} \in \mathscr{R}$. Since $\lambda \neq \mathfrak{s}$ and $u_{2}^{1} \notin \mathscr{R}$, this yields $\lambda=\mu$. Thus we have shown that $\mathscr{R}_{12}=\mathscr{B}_{+, z}$ by Lemma 3.3. Consequently, $\mathscr{R}=\mathscr{R}_{+, z}$.

The remaining parts of the proofs are very similar to the proofs given in [SS2], Sect. 4. We shall indicate only a few points where some modifications are necessary. Put $q_{+}:=q^{-1}$ and $q_{-}:=-q^{-2 N-1}$. Setting $\omega_{i j}:=z Q q_{ \pm}^{-1}\left(X_{ \pm, z}\right)_{i j}^{n m} \eta_{n m}$, then we obtain $\mathrm{d} u_{j}^{n}=u_{i}^{n} \omega_{i j}$ by the same computation as in [SS2]. Since $z \mathfrak{r}_{ \pm} \neq \mathfrak{s}$ by assumption, the linear mapping $X_{ \pm, z}$ is invertible and hence $\Gamma_{ \pm, z}=\left\langle a \cdot \mathrm{d} u_{j}^{i}\right\rangle$. As in [SS2], the pair $\left(\Gamma_{ \pm, z}, \mathrm{~d}\right)$ is a bicovariant differential calculus over $\mathscr{A}$ and it is of the form described in Theorem 2.1, (iii). The formulas for $\mathrm{d} \mathscr{D}$ and $\mathrm{d} x$ follow at once from the relations $\omega=\left(z \mathfrak{x}_{ \pm}-\mathfrak{s}\right) \eta$ and $\eta \mathscr{D}=z^{N} q^{ \pm 2} \mathscr{D} \eta$ which are easily verified.

Next we show that for $N \geqq 3$ the bicovariant differential calculus $\left(\Gamma_{ \pm, z}, \mathrm{~d}\right)$ is associated with the ad-invariant right ideal $\mathscr{R}_{ \pm, z}$. Let ${ }_{z}^{ \pm} \chi_{n m}$ denote the linear functionals $\chi_{n m}$ (see Theorem 2.1, (iii)) for the calculus $\left(\Gamma_{ \pm, z}, \mathrm{~d}\right.$ ). Fix a pair $\left(\Gamma_{\tau, z}, \mathrm{~d}\right)$ with $\tau \in\{+,-\}, z \neq 0$ and $z \mathfrak{r}_{\tau} \neq \mathfrak{s}$. From the general theory [W] it follows that its associated ad-invariant right ideal $\mathscr{R}$ has the properties stated in Theorem 2.1, (i). Therefore, by the above proof, $\mathscr{R}$ is equal to some right ideal $\mathscr{R}_{\tau^{\prime}, z^{\prime}}$. We have to show that $(\tau, z)=\left(\tau^{\prime}, z^{\prime}\right)$. We restrict ourselves to the case $\tau^{\prime}=+$. The case $\tau^{\prime}=-$ is treated in a similar manner. As in [SS2], we consider the elements $a_{i j}:=\left(U-z^{\prime} \mathbf{r}_{+}\right) u_{j}^{i}=\left(v_{+}\right)_{j}^{i}+\left(v_{-}\right)_{J}^{i}-\left(\lambda_{+, z^{\prime}}^{+}+\lambda_{+, z}^{-}\right) u_{j}^{i}$ and $b_{i j}:=v_{j}^{i}-z^{\prime}\left(q+q^{-3}\right) u_{j}^{i}=q\left(v_{+}\right)_{j}^{i}-q^{-1}\left(v_{-}\right)_{j}^{l}-\left(q \lambda_{+, z}^{+},-q^{-1} \lambda_{+, z \prime}^{-}\right) u_{j}^{i}$ for $i \neq j$ of the right ideal $\mathscr{R}_{+, z}$, and we compute

$$
\begin{aligned}
& { }_{z}^{-} \chi_{i j}\left(a_{i j}\right)=z \mathfrak{r}_{-}-z^{\prime} \mathfrak{r}_{+},{ }_{z}^{-} \chi_{i j}\left(b_{i j}\right)=z\left(q^{-1}+q^{-2 N-1}\right)-z^{\prime}\left(q+q^{-3}\right) \text { and } \\
& { }_{z}^{+} \chi_{i j}\left(b_{i j}\right)=\left(z-z^{\prime}\right)\left(q+q^{-3}\right) .
\end{aligned}
$$

Recall that again by the general theory [W], the functionals ${ }_{z}^{\tau} \chi_{i j}$ annihilate the right ideal $\mathscr{R}_{+, z^{\prime}}$ which is associated with the bicovariant calculus $\left(\Gamma_{\tau, z}, \mathrm{~d}\right)$. Therefore, if $\tau$ would be - , then we have $z \mathfrak{r}_{-}=z^{\prime} \mathfrak{r}_{+}$and $z\left(q^{-1}+q^{-2 N-1}\right)=z^{\prime}\left(q+q^{-3}\right)$ by (3.6). Some computations show that the two latter equations lead to a contradiction if $q$ is not a root of unity. Hence $\tau=+$. From ${ }_{z}^{+} \chi_{i j}\left(b_{i j}\right)=0$ we obtain $z=z^{\prime}$, so that $(\tau, z)=\left(\tau^{\prime}, z^{\prime}\right)$. In the case $N=2$ the elements $a_{i j}=\left(U-z^{\prime} \mathfrak{r}_{+}\right) u_{j}^{i}, i \neq j$, belong to $\mathscr{R}_{+, z}$ and the preceding arguments prove that the bicovariant differential calculus $\left(\Gamma_{+, z}, \mathrm{~d}\right)$ is associated with the right ideal $\mathscr{R}_{+, z}$. Recall that for $N=2$ each adinvariant right ideal $\mathscr{R}$ with $\operatorname{ker} \varepsilon=\mathscr{R}+\tilde{\mathscr{A}}_{1}$ and codim $\mathscr{R} \geqq 2$ is equal to some $\mathscr{R}_{+, z}$.

Finally, let us note that all right ideals $\mathscr{R}_{ \pm, z}$ for $N \geqq 3$ and $\mathscr{R}_{+, z}$ for $N=2$ are different, hence the corresponding differential calculi $\left(\Gamma_{ \pm, z}, \mathrm{~d}\right)$ for $N \geqq 3$ and $\left(\Gamma_{+, z}, \mathrm{~d}\right)$ for $N=2$ are non-isomorphic.

This completes the proofs of Theorems 2.1 and 2.2. 


\section{Bicovariant Differential Calculi on $O_{q}(N)$ and $S p_{q}(N)$ (Revised)}

From now on let $\mathscr{A}$ be the Hopf algebra of functions for one of the quantum groups $O_{q}(N)$ or $S p_{q}(N)$ with $N \geqq 3$. We keep all notations and abbreviations from Part II of our previous paper [SS2].

Let $\mathscr{R}_{ \pm}$be the right ideals of ker $\varepsilon$ and $\left(\Gamma_{ \pm}, \mathrm{d}\right)$ the bicovariant differential calculi on $\mathscr{A}$ defined in [SS2], Sect. 6. Recall from [SS2] that $\mathscr{R}_{-}$and $\left(\Gamma_{-}, \mathrm{d}\right)$ are only defined if the number $Q Z+2 \mathfrak{s}$ does not vanish, where $Q:=q-q^{-1}, Z:=q^{N-1}-$ $q^{1-N}$ for $\mathscr{A}=O_{q}(N), Z:=q^{-N-1}-q^{N+1}$ for $\mathscr{A}=S p_{q}(N)$, and $\mathfrak{s}:=1+Z Q^{-1}$.

The following theorem is a strengthening of Theorems 6.1 and 6.2 in [SS2].

Theorem 4.1. Suppose that $N \geqq 4$. Let $\mathscr{A}=O_{q}(N)$ or $\mathscr{A}=S p_{q}(N)$. The right ideals $\mathscr{R}_{+}$and $\mathscr{R}_{-}$for $Q Z+2 \mathfrak{s} \neq 0$ are the only ad-invariant right ideals $\mathscr{R}$ of ker $\varepsilon$ such that codim $\mathscr{R} \geqq 2$ and $\operatorname{ker} \varepsilon=\mathscr{R}+\tilde{\mathscr{A}}_{1}$. The differential calculi $\left(\Gamma_{+}, \mathrm{d}\right)$ and $\left(\Gamma_{-}, \mathrm{d}\right)$ for $Q Z+2 \mathfrak{s} \neq 0$ are the only bicovariant differential calculi $(\Gamma, \mathrm{d})$ over $\mathscr{A}$ for which $\Gamma=\left\langle a \mathrm{~d} u_{j}^{i}: a \in \mathscr{A}, i, j=1, \ldots, N\right\rangle$ and $\operatorname{dim}(\Gamma, \mathrm{d}) \geqq 2$.

In order to formulate the corresponding result for $\mathscr{A}=O_{q}(3)$, we need to define two new right ideals $\mathscr{R}_{1}$ and $\mathscr{R}_{2}$ of ker $\varepsilon$ and two new bicovariant differential calculi $\left(\Gamma_{1}, \mathrm{~d}\right)$ and $\left(\Gamma_{2}, \mathrm{~d}\right)$ on $O_{q}(3)$.

For $k=1,2$, we set $\mathscr{R}_{k}:=\mathscr{B}_{k} \cdot \mathscr{A}$, where $\mathscr{B}_{k}$ is the linear span of the following elements:

(1) $S_{+}\left(u_{j}^{i} u_{m}^{n}\right)$ for $i, j, n, m=1, \ldots, N$.

(2) $\left(f_{+}\right)_{j}^{i},\left(f_{-}\right)_{j}^{i},\left(u_{+}\right)_{j}^{i},\left(g_{+}\right)_{j}^{i}-\psi_{k}^{+}\left(u_{-}\right)_{j}^{i}$ and $\left(g_{-}\right)_{j}^{i}-\psi_{k}^{-}\left(u_{-}\right)_{j}^{i}$ for $i, j=1, \ldots, N$.

(3) $\tilde{V}_{+}-\mu_{k}^{+} \tilde{U}, \tilde{V}_{-}-\mu_{k}^{-} \tilde{U}$.

Here the coefficients $\psi_{k}^{ \pm}$and $\mu_{k}^{ \pm}$are given by

$$
\begin{aligned}
& \psi_{1}^{+}=-\psi_{2}^{+}:=\left(1-Q_{+}^{-1}\right)\left(q^{2}+q+1+q^{-1}+q^{-2}\right), \psi_{1}^{-}=-\psi_{2}^{-}:=1-Q_{+}^{-1}, \\
& \mu_{1}^{+}:=q^{2}+q+1+q^{-1}+q^{-2}, \mu_{1}^{-}:=1 \text { and } \mu_{2}^{ \pm}:=-(q-1)^{2}\left(q^{2}+1\right)^{-1} \mu_{1}^{ \pm} .
\end{aligned}
$$

To define the two new differential calculi on $O_{q}(3)$, some preliminaries are necessary. For this we consider the quantum group $S L_{p}(2)$, where the complex number $p$ is chosen such that the square root of $q$ appearing in the matrix $C$ of the metric for $O_{q}(3)$ is equal to $-p$. That is, the elements in the offdiagonal of $C$ are $-p^{-1}, 1,-p$ and we have $p^{2}=q$. We denote the fundamental matrix of $S L_{p}(2)$ by $t=\left(t_{j}^{i}\right)_{i, j=1,2}$.

There exists a Hopf algebra morphism $\pi: O_{q}(3) \rightarrow S L_{p}(2)$ such that

$$
\left(\pi\left(u_{j}^{i}\right)\right)_{i, J=1,2,3}=\left(\begin{array}{ccc}
\left(t_{1}^{1}\right)^{2} & \left(1+p^{2}\right)^{1 / 2} t_{2}^{1} t_{1}^{1} & \left(t_{2}^{1}\right)^{2} \\
\left(1+p^{2}\right)^{1 / 2} t_{1}^{2} t_{1}^{1} & 1+\left(p+p^{-1}\right) t_{2}^{1} t_{1}^{2} & \left(1+p^{2}\right)^{1 / 2} t_{2}^{2} t_{2}^{1} \\
\left(t_{1}^{2}\right)^{2} & \left(1+p^{2}\right)^{1 / 2} t_{2}^{2} t_{1}^{2} & \left(t_{2}^{2}\right)^{2}
\end{array}\right) .
$$

(Here the choice of the square root for $1+p^{2}$ is always the same, but arbitrary.) To prove this, one verifies by direct computations that the above matrix entries satisfy all relations of the definition of $O_{q}(3)$. For this it is essential that $q^{1 / 2}=-p$. Therefore, the map $u_{j}^{i} \rightarrow \pi\left(u_{j}^{i}\right)$ extends uniquely to a unital algebra homomorphism $\pi$ of $O_{q}(3)$ into $S L_{p}(2)$. From the construction it is clear that $\pi$ is a Hopf algebra morphism. Since obviously $\left\langle\mathbb{1}, \pi\left(u_{j}^{l}\right): i, j=1,2,3\right\rangle=\left\langle\mathbb{1}, t_{m}^{n} t_{s}^{r}: n, m, r, s=1,2\right\rangle$, we 
see that the image $\pi\left(O_{q}(3)\right)$ is precisely the unital subalgebra of $S L_{p}(2)$ which is generated by all quadratic monomials $t_{m}^{n} t_{s}^{r}$. Note that above matrix $\left(\pi\left(u_{j}^{i}\right)\right)$ is nothing but the matrix for the spin 1 representation of the quantum group $S L_{p}(2)$. See, for instance, formula $(2.13)$ in $[D K]$. Let $(\tilde{\Gamma}, \mathrm{d})$ denote the $4 D_{+}$-calculus on $S L_{p}(2)$ invented by S.L. Woronowicz [W]. Recall that this calculus is characterized in [W] by its associated ad-invariant right ideal $\mathscr{S}_{+}$of ker $\varepsilon$. That is, $\mathscr{S}_{+}$is the right ideal of ker $\varepsilon$ which is generated by the elements

$$
\begin{aligned}
& \left(t_{1}^{1}\right)^{2}+p^{2}\left(t_{2}^{2}\right)^{2}-\left(1+p^{2}\right)\left(t_{1}^{1} t_{2}^{2}+p^{-1} t_{2}^{1} t_{1}^{2}\right),\left(t_{2}^{1}\right)^{2},\left(t_{1}^{2}\right)^{2}, t_{2}^{1}\left(t_{1}^{1}-t_{2}^{2}\right), \\
& t_{1}^{2}\left(t_{1}^{1}-t_{2}^{2}\right),(T-\gamma) t_{2}^{1},(T-\gamma) t_{1}^{2},(T-\gamma)\left(t_{1}^{1}-t_{2}^{2}\right),(T-\gamma)\left(T-1-p^{2}\right),
\end{aligned}
$$

where $T:=p^{2} t_{1}^{1}+t_{2}^{2}$ and $\gamma:=p^{-1}+p^{-3}$. (Note that in [W], p. 132, Example 3, only the quantum group $S U_{p}(2)$ was considered, but the generalization to $S L_{p}(2)$ is straightforward.)

The most convenient and easiest way for our purposes to describe the calculus $(\tilde{\Gamma}, \mathrm{d})$ on $S L_{p}(2)$ and to construct the new calculi $\left(\Gamma_{k}, \mathrm{~d}\right), k=1,2$, on $O_{q}(3)$ is to use the method of B. Jurčo [Ju]. In fact, $(\tilde{\Gamma}, \mathrm{d})$ has the structure of the calculus $\left(\Gamma_{+, z}, \mathrm{~d}\right)$ defined in Sect. 2 with $z=p^{-1}$ and $G L_{q}(N)$ replaced with $S L_{p}(2)$. That is, the bicovariant bimodule $\tilde{\Gamma}$ is of the form $\left(t^{c} \otimes t, L^{+} \otimes L^{-, c}\right)$ and the mapping $\mathrm{d}: S L_{p}(2) \rightarrow \tilde{\Gamma}$ given by $\mathrm{d} x=\eta x-x \eta$ for $x \in S L_{p}(2)$, where $\eta:=p^{-2} \eta_{11}+$ $p^{-4} \eta_{22}=q^{-1} \eta_{11}+q^{-2} \eta_{22}$ and $\left\{\eta_{l j}: i, j=1,2\right\}$ is the corresponding basis of $\tilde{\Gamma}_{l n v}$. Recall that $t^{c}$ denotes the contragredient representation of the fundamental representation of $S L_{p}(2)$.

The map $g:=\left(L^{+} \otimes L^{-, c}\right) \pi$ is a homomorphism of the algebra $O_{q}(3)$ into $M_{4}(\mathbb{C})$. That is, we have $g=\left(g_{r s}^{n m}\right)$ and $g_{r s}^{n m}(a)={ }^{+} l_{r}^{n}\left(\pi(a)_{(1)}\right) \kappa\left({ }^{-} l_{m}^{s}\right)\left(\pi(a)_{(2)}\right)$ for $n, m, r, s=1,2$ and $a \in O_{q}(3)$ with $\Delta(a)=a_{(1)} \otimes a_{(2)}$. Let $\Gamma_{1}$ be the free left module for $\mathscr{A}=O_{q}(3)$ with basis $\left\{\eta_{r s}: r, s=1,2\right\}$. There is a unique $\mathscr{A}$-bimodule structure on $\Gamma_{1}$ such that $\eta_{r s} a=\left(g_{r s}^{n m} * a\right) \eta_{n m}, a \in O_{q}(3)$. We define d $a:=\eta a-a \eta$ for $a \in O_{q}(3)$. Then the pair $\left(\Gamma_{1}, \mathrm{~d}\right)$ is a bicovariant differential calculus over $\mathscr{A}=O_{q}(3)$. From the preceding definition we conclude easily that there exists a well-defined linear mapping $\Psi: \Gamma_{1} \rightarrow \Gamma_{+, z}$ such that $\Psi\left(a \eta_{i j} b\right)=\pi(a) \eta_{i j} \pi(b)$ and $\Psi(a \mathrm{~d} b)=\pi(a \mathrm{~d} \pi(b))$ for $a, b \in O_{q}(3)$ and $i, j=1,2$.

Being a bicovariant bimodule, $\Gamma_{1}$ can be written as a pair $(w, g)$, where $w=\left(w_{r s}^{n m}\right)$ is a representation of the coalgebra $O_{q}(3)$, cf. [W], Theorems 2.3 and 2.4 , or [SS2], p. 638. The matrix entries $w_{r s}^{n m}$ are the (uniquely determined) elements in the linear span $\left\langle u_{j}^{i}, \mathbb{1}: i, j=1,2,3\right\rangle$ for which $\pi\left(w_{r s}^{n m}\right)=\kappa\left(t_{n}^{r}\right) t_{s}^{m}$. For explicit computations it is convenient to write $w$ as a direct sum [2] $\oplus[0]$ and to use the matrix representations $u_{-}=\left(\left(u_{-}\right)_{j}^{i}\right)$ for the Young tableau [2] and $\tilde{U}$ for the trivial representation [0].

Since all defining relations for $O_{q}(3)$ are quadratic in the matrix entries $u_{j}^{i}$, there are well-defined linear functionals $h_{r s}^{n m}$ on $O_{q}(3)$ such that $h_{r s}^{n m}(\mathbb{1})=\delta_{n r} \delta_{m s}$ and $h_{r s}^{n m}(a)=(-1)^{k} g_{r s}^{n m}(a)$ for $a \in \mathscr{A}_{k}$. The pair $(w, h)$ with $h=\left(h_{r s}^{n m}\right)$ is also a bicovariant bimodule for $O_{q}(3)$ which is denoted by $\Gamma_{2}$. As in case of $\Gamma_{1}$ we set $\mathrm{d} a:=\eta a-a \eta$ for $a \in O_{q}(3)$ and the pair $\left(\Gamma_{2}, \mathrm{~d}\right)$ is a bicovariant differential calculus over $\mathscr{A}=O_{q}(3)$. (The missing details of proof for both $\left(\Gamma_{1}, \mathrm{~d}\right)$ and $\left(\Gamma_{2}, \mathrm{~d}\right)$ will be provided at the end of Sect. 5.) Obviously, the calculi $\left(\Gamma_{k}, \mathrm{~d}\right), k=1,2$, are four dimensional and inner. 
Our classification theorem for the quantum group $O_{q}(3)$ can be now stated as follows.

Theorem 4.2. Suppose that $\mathscr{A}$ is the quantum group $O_{q}(3)$. The four right ideals $\mathscr{R}_{+}, \mathscr{R}_{-}, \mathscr{R}_{1}$ and $\mathscr{R}_{2}$ are the only ad-invariant right ideals $\mathscr{R}$ of ker $\varepsilon$ with codim $\mathscr{R} \geqq 2$ and ker $\varepsilon=\mathscr{R}+\tilde{A}_{1}$. The pairs $\left(\Gamma_{+}, \mathrm{d}\right),\left(\Gamma_{-}, \mathrm{d}\right),\left(\Gamma_{1}, \mathrm{~d}\right)$ and $\left(\Gamma_{2}, \mathrm{~d}\right)$ are the only bicovariant differential calculi $(\Gamma, \mathrm{d})$ over $\mathscr{A}$ for which $\Gamma=\left\langle a \mathrm{~d} u_{j}^{i}: a \in \mathscr{A}, i, j=1,2,3\right\rangle$ and $\operatorname{dim}(\Gamma, \mathrm{d}) \geqq 2$. The pair $\left(\Gamma_{\tau}, \mathrm{d}\right)$ is the canonical calculus associated with the ad-invariant right ideal $\mathscr{R}_{\tau}$ for $\tau=+,-, 1,2$.

Before we begin the rather long proofs of Theorems 4.1 and 4.2, we shall study the two new bicovariant differential calculi $\left(\Gamma_{k}, \mathrm{~d}\right), k=1,2$, over $\mathscr{A}=O_{q}(3)$ in more detail. For this we take Theorem 4.2 for granted and we fix $k \in\{1,2\}$.

First we shall describe these calculi explicitly in terms of a basis of Maurer-Cartan forms. (For the calculi $\left(\Gamma_{ \pm}, \mathrm{d}\right)$ over $O_{q}(N)$ and $S p_{q}(N)$ this is done in Theorem 6.2 of [SS2]. The bimodule structure of $\Gamma_{ \pm}$and the differentiation d are expressed with respect to the Maurer-Cartan basis $\left\{\omega_{i j}\right\}$ by the linear transformation $T_{ \pm}$defined in [SS2], Sect. 6.) We define a basis $\left\{\omega^{1}, \omega^{2}, \omega^{3}, \omega^{4}\right\}$ of the vector space $\left(\Gamma_{k}\right)_{i n v}$ of left-invariant forms of $\Gamma_{k}$ by setting

$$
\omega^{1}:=P_{i n v}\left(\mathrm{~d}\left(u_{-}\right)_{1}^{1}\right), \omega^{2}:=P_{i n v}\left(\mathrm{~d}\left(u_{-}\right)_{2}^{1}\right), \omega^{3}:=P_{i n v}\left(\mathrm{~d}\left(u_{-}\right)_{1}^{2}\right), \omega^{4}=\omega:=P_{i n v}(\mathrm{~d} \tilde{U}) .
$$

Since ker $\varepsilon=\mathscr{R}_{k}+\left\langle\left(u_{-}\right)_{j}^{i}: i, j=1,2,3\right\rangle+\langle\tilde{U}\rangle$ by the definition of $\mathscr{R}_{k}$ and codim $\mathscr{R}_{k}=\operatorname{dim}\left(\Gamma_{k}, \mathrm{~d}\right)=4$, this set is indeed a vector space basis of $\left(\Gamma_{k}\right)_{i n v}$; cf. the proof of Lemma 1.5 in [SS2]. Put $\psi_{k}:=\psi_{k}^{+}+\psi_{k}^{-}$. Using the definition of $\pi(U)=q \pi\left(u_{1}^{1}\right)+\pi\left(u_{2}^{2}\right)+q^{-1} \pi\left(u_{3}^{3}\right)$ and the commutation rules of the bimodule $\Gamma_{+, z}$ for $S L_{p}(2)$ we compute $\omega=P_{\text {inv }}(d \tilde{U})=\left(\psi_{k}-\mathfrak{s}\right) \eta$. (For $k=2$ this follows also from the facts that $\tilde{\mathscr{D}}+2\left(\psi_{2}-\mathfrak{s}\right)^{-1} \tilde{U} \in \mathscr{R}_{2}$ and $\mathrm{d} \tilde{\mathscr{D}}=-2 \mathscr{D} \eta$ which are proved in Sect. 5.) Thus the differentiation $\mathrm{d}$ of the calculus $\left(\Gamma_{k}, \mathrm{~d}\right)$ can be written as

$$
\mathrm{d} a=\left(\psi_{k}-s\right)^{-1}(\omega a-a \omega) \text { for } a \in O_{q}(3) \text {. }
$$

Let $A=\left(A_{i j}^{n}\right)_{i j=1,2,3 ; n=1, \ldots, 4}$ be the matrix of $\operatorname{Mor}\left(u^{c} \otimes u,[2] \oplus[0]\right)$ defined by $A_{11}^{1}=$ $A_{12}^{3}:=1, A_{11}^{4}=A_{22}^{4}=A_{33}^{4}:=\mathfrak{s}^{-1}, A_{22}^{1}:=1-q, A_{23}^{2}=A_{32}^{3}:=-q^{-1 / 2}$ and $A_{i j}^{n}:=0$ otherwise. Then we have

$$
\mathrm{d} u_{j}^{i}=u_{l}^{i} A_{l j}^{n} \omega^{n} \text { for } i, j=1,2,3 .
$$

The right action $\Delta_{R}$ of $O_{q}(3)$ on the basis elements $\omega^{n}$ is given by

$$
\Delta_{R}\left(\omega^{n}\right)=\omega^{m} \otimes v_{n}^{m},
$$

where the elements $v_{n}^{m}$ of $\mathscr{A}_{1}+\mathbb{C} \cdot \mathbb{1}$ are characterized by the equations $A_{i j}^{n} \kappa\left(u_{i}^{k}\right) u_{l}^{j}=$ $v_{m}^{n} A_{k l}^{m}$ for $n=1, \ldots, 4$ and $k, l=1,2,3$. In fact, $\left(v_{n}^{m}\right)$ is the matrix for the subrepresentation [2] $\oplus[0]$ of $u^{c} \otimes u$ with respect to the basis $\left\{\omega^{1}, \omega^{2}, \omega^{3}, \omega^{4}\right\}$.

Let $\left\{\omega_{1}, \omega_{2}, \omega_{3}, \omega_{4}\right\}$ denote the basis of $\left(\Gamma_{k}\right)_{i n v}$ defined by

$$
\begin{aligned}
& \omega_{1}:=\omega^{1}+(q-1)\left(Q_{+}+1\right) \omega^{4}, \omega_{2}:=\omega^{2}, \\
& \omega_{3}:=\omega^{3}, \omega_{4}:=\left(Q_{+}+1\right) \omega^{4} \text { for } k=1
\end{aligned}
$$

and $\omega_{1}:=-\omega^{1}-q Q_{+}\left(Q_{+}+1\right)(q-1)^{-1} \omega^{4}, \omega_{2}:=-\omega^{2}, \omega_{3}:=-\omega^{3}$, $\omega_{4}:=q Q_{+}\left(Q_{+}+1\right)(q-1)^{-2} \omega^{4}$ for $k=2$. 
Then the bimodule structure of $\Gamma_{k}$ can be expressed as

$$
\omega_{n} a=\left(f_{m}^{n} * a\right) \omega_{m} \text { for } a \in O_{q}(3) \text { and } n=1, \ldots, 4
$$

where the $f=\left(f_{m}^{n}\right): O_{q}(3) \rightarrow M_{4}(\mathbb{C})$ is the unital algebra homomorphism given by the following formulas:

$$
\begin{aligned}
& f\left(u_{1}^{1}\right)= \pm \operatorname{diag}\left(q, 1,1, q^{-1}\right) \pm e_{14}, f\left(u_{2}^{2}\right)= \pm \operatorname{diag}(1,1,1,1) \pm\left(1-q^{-1}\right) e_{14}, \\
& f\left(u_{3}^{3}\right)= \pm \operatorname{diag}\left(q^{-1}, 1,1, q\right) \mp q^{-1} e_{14}, f\left(u_{3}^{1}\right)=f\left(u_{1}^{3}\right)=0, \\
& f\left(u_{2}^{1}\right)= \pm\left(1-q^{-1}\right) e_{12} \pm e_{34}, f\left(u_{1}^{2}\right)= \pm\left(1-q^{-1}\right) e_{13} \pm e_{24}, \\
& f\left(u_{3}^{2}\right)=\mp q^{-1 / 2}\left(1-q^{-1}\right) e_{12} \mp q^{1 / 2} e_{34}, f\left(u_{2}^{3}\right)=\mp q^{-1 / 2}\left(1-q^{-1}\right) e_{13} \mp q^{1 / 2} e_{24} .
\end{aligned}
$$

Here diag $(\ldots)$ is the corresponding diagonal matrix and $e_{n m}$ denotes the matrix with 1 in the $(n, m)$ position and 0 elsewhere. The upper sign refers to $k=1$ and the lower sign to $k=2$.

Let $\mathscr{D}$ denote the quantum determinant for the quantum group $O_{q}(3)$, see e.g. [T and $\mathrm{H}$ ]. For $O_{q}(3)$ the completely antisymmetric tensor $\left(\varepsilon_{i j k}\right)$ is given by $\varepsilon_{123}=1, \varepsilon_{132}=\varepsilon_{213}=-\varepsilon_{312}=-\varepsilon_{231}=-q, \varepsilon_{321}=-q^{2}, \varepsilon_{222}=-q\left(q^{1 / 2}-q^{-1 / 2}\right)$ and zero otherwise, so that

$$
\begin{aligned}
\mathscr{D}= & u_{1}^{1} u_{2}^{2} u_{3}^{3}+q u_{3}^{1} u_{1}^{2} u_{2}^{3}+q u_{2}^{1} u_{3}^{2} u_{1}^{3}-q u_{2}^{1} u_{1}^{2} u_{3}^{3}-q u_{1}^{1} u_{3}^{2} u_{2}^{3} \\
& -q\left(q^{1 / 2}-q^{-1 / 2}\right) u_{2}^{1} u_{2}^{2} u_{2}^{3}-q^{2} u_{3}^{1} u_{2}^{2} u_{1}^{3} \\
= & u_{1}^{1} u_{2}^{2} u_{3}^{3}+\left(q+q^{2}\right) u_{2}^{1} u_{3}^{2} u_{1}^{3}-(1+q) u_{1}^{1} u_{3}^{2} u_{2}^{3}-q^{2} u_{3}^{1} u_{2}^{2} u_{1}^{3}
\end{aligned}
$$

where the second equality follows from the first one by the commutation rules for the matrix entries $u_{j}^{i}$. The quantum determinant $\mathscr{D}$ is group-like, it is central in the algebra $O_{q}(3)$ and its square is $\mathbb{1}$. The quotient of $O_{q}(3)$ by the two-sided ideal generated by $\mathscr{D}-\mathbb{1}$ becomes again a Hopf algebra which is called the quantum group $\mathrm{SO}_{q}(3)$.

From the definition of $\pi$ and (4.1) we compute $\pi(\mathscr{D}-\mathbb{1})=0$. Therefore, the calculus $\left(\Gamma_{1}\right.$, d) factorizes through $S_{q}(3)$ and defines a bicovariant differential calculus over the quantum group $\mathrm{SO}_{q}(3)$. It can be shown that the kernel of the Hopf algebra morphism $\pi: O_{q}(3) \rightarrow S L_{p}(2)$ coincides with the two-sided ideal generated by the element $\mathscr{D}-\mathbb{1}$, so the Hopf algebra $S O_{q}(3)$ is isomorphic to a Hopf subalgebra of $S L_{p}(2)$. Clearly, the latter is the algebra generated by $\mathbb{1}$ and all quadratic terms $t_{m}^{n} t_{s}^{r}$. Identifying $S O_{q}(3)$ with this Hopf subalgebra, the bicovariant calculus $\left(\Gamma_{1}, \mathrm{~d}\right)$ over $S O_{q}(3)$ is just the restriction to $S O_{q}(3)$ of the $4 D_{+}$-calculus over $S L_{p}(2)$.

For the second calculus $\left(\Gamma_{2}, \mathrm{~d}\right)$ we have $\mathrm{d} \mathscr{D}=-2 \mathscr{D} \eta=-2\left(\psi_{2}-\mathfrak{s}\right)^{-1} \mathscr{D} \omega \neq 0$. Hence the calculus $\left(\Gamma_{2}, \mathrm{~d}\right)$ does not factorize through $\mathrm{SO}_{q}(3)$.

\section{Remarks.}

1) In the preceding we could have replaced the $4 D_{+}$-calculus on $S L_{p}(2)$ with the $4 D_{-}$-calculus.

2) It can be shown that $\mathscr{R}_{1}=\left\{a \in \operatorname{ker} \varepsilon: \pi(a) \in \mathscr{S}_{+}\right\}=\left\{a \in \operatorname{ker} \varepsilon: \pi(a) \in \mathscr{S}_{-}\right\}$, where $\mathscr{S}_{ \pm}$is the ad-invariant right ideal of ker $\varepsilon$ associated with the $4 D_{ \pm}$-calculus. 


\section{Proofs of Theorems 4.1 and 4.2}

Let $\mathscr{A}$ be one of the quantum groups $O_{q}(N)$ or $S p_{q}(N)$ with $N \geqq 3$. Suppose that $\mathscr{R}$ is an ad-invariant right ideal of ker $\varepsilon$ such that ker $\varepsilon=\mathscr{R}+\tilde{\mathscr{A}}_{1}$ and codim $\mathscr{R} \geqq 2$. Set $\mathscr{R}_{12}:=\mathscr{R}_{1} \cap\left(\tilde{\mathscr{A}_{1}}+\tilde{\mathscr{A}}_{2}\right)$.

Lemma 5.1. There are complex numbers $\varphi^{+}, \varphi^{-}, \psi^{+}, \psi^{-}, \mu^{+}, \mu^{-}$and numbers $\delta_{0}, \delta_{+}, \delta_{-} \in\{0,1\}$ with $\delta_{+} \delta_{-}=0$ such that

$$
\begin{aligned}
\mathscr{R}_{12}= & \delta_{0}\langle\tilde{U}\rangle \oplus \mathscr{H}_{+} \oplus \mathscr{H}_{-} \oplus \delta_{+}\left\langle u_{+}\right\rangle \oplus \delta_{-}\left\langle u_{-}\right\rangle \oplus \mathscr{F}_{+} \oplus \\
& \mathscr{F}_{-} \oplus \mathscr{G}_{+} \oplus \mathscr{G}_{-} \oplus \operatorname{im} S_{+} \oplus \operatorname{im} S_{-},
\end{aligned}
$$

where $\mathscr{H}_{ \pm}:=\left\langle\tilde{V}_{ \pm}-\mu^{ \pm} \tilde{U}\right\rangle,\left\langle u_{ \pm}\right\rangle:=\left\langle\left(u_{ \pm}\right)_{j}^{i}\right\rangle, \mathscr{F}_{ \pm}:=\left\langle\left(f_{ \pm}\right)_{j}^{l}-\varphi^{ \pm}\left(u_{+}\right)_{j}^{i}\right\rangle$ and $\mathscr{G}_{ \pm}:=$ $\left\langle\left(g_{ \pm}\right)_{j}^{l}-\psi^{ \pm}\left(u_{-}\right)_{j}^{i}\right\rangle$. We have $\mathscr{G}_{-}=\{0\}$ (that is, $\left(g_{-}\right)_{j}^{i}=0$ for all $i, j$ and $\psi^{-}=0$ ) for $\mathscr{A}=S p_{q}(4)$ and $S_{-}=0$ for $\mathscr{A}=O_{q}(3)$.

Proof. The proof is similar to the proof of Lemma 8.2 in [SS2]. By the proof given there, we obtain for $\mathscr{A} \neq S p_{q}(4)$ and $\mathscr{A} \neq O_{q}(3)$ the following decomposition:

$$
\begin{aligned}
\operatorname{ad}\left[\mathscr{R}_{12} \cong\right. & \left(2+\delta_{0}\right)[0] \oplus\left(2+\delta_{+}\right)[2] \oplus\left(2+\delta_{-}\right)\left[1^{2}\right] \oplus([2,2] \oplus[3,1] \oplus[4]) \\
& \oplus\left([2,2] \oplus[2,1,1] \oplus\left[1^{4}\right]\right)
\end{aligned}
$$

with $\delta_{0}, \delta_{+}, \delta_{-} \in\{0,1\}$. Note that the summand $\left[1^{4}\right]$ in (5.2) vanishes for $\mathscr{A}=$ $S p_{q}(6)$. (Recall we assumed in [SS2] that ker $\varepsilon=\mathscr{R} \oplus \tilde{\mathscr{A}_{1}}$, so that $\delta_{0}=\delta_{+}=\delta_{-}=$ 0 therein.) We have $\delta_{+}=0$ or $\delta_{-}=0$, since otherwise codim $\mathscr{R} \leqq 1$. As in [SS2], the corresponding subspaces of the decomposition (5.2) can be written of the form (5.1). If $\delta_{0}=1$ (resp. $\left.\delta_{+}=1, \delta_{-}=1\right)$, we set $\mu^{ \pm}=0$ (resp. $\left.\varphi^{ \pm}=0, \psi^{ \pm}=0\right)$.

For $\mathscr{A}=S p_{q}(4)$ the summands $[2,2,1]$ and $\left[1^{4}\right]$ in (5.2) vanish, the coefficient of $\left[1^{2}\right]$ is $1+\delta_{-}$, and $\left(g_{-}\right)_{j}^{i}=0$ for all $i, j=1, \ldots, N$. Setting $\psi^{-}=0$, (5.1) still holds in this case. For $\mathscr{A}=O_{q}(3)$, the summands [2,2], [2,1,1],[1 $]$ disappear and $S_{-}=0$, so $(5.1)$ is also valid.

First we treat the case $\delta_{+}=\delta_{-}=0$. This assumption was sufficient to derive the eight equations of Lemmas 8.4 and 8.5 in [SS2]. (Note that the condition $\delta_{0}=0$ was not needed for this.) From these equations the values of $\varphi^{ \pm}, \psi^{ \pm}$and $\mu^{ \pm}$were computed. In particular, it was shown that either $\varphi^{+}+\varphi^{-}-\mathfrak{s}=Q Z \neq 0$ or $\varphi^{+}+\varphi^{-}-\mathfrak{s}=-Q Z-\mathfrak{s} \neq 0$. If $\delta_{0}=1$, then $\tilde{U} \in \mathscr{R}$ and thus $0 \equiv \tilde{U} u_{1^{\prime}}^{1} \equiv\left(\varphi^{+}+\right.$ $\left.\varphi^{-}-\mathfrak{s}\right) u_{1^{\prime}}^{1}$, so that $\varphi^{+}+\varphi^{-}-\mathfrak{s}=0$, since $\delta_{+}=\delta_{-}=0$, and hence $u_{1^{\prime}}^{1} \notin \mathscr{R}$. This is a contradiction, which proves that $\delta_{0}=0$. Therefore, ker $\varepsilon=\mathscr{R} \oplus \tilde{\mathscr{A}_{1}}$ and hence $\mathscr{R}=\mathscr{R}_{+}$or $\mathscr{R}=\mathscr{R}_{-}$by the proof of Theorem 6.1 in [SS2].

The other possible cases require a number of additional lengthy calculations. For this we freely use the abbreviations $a_{ \pm}, a^{ \pm}, b_{ \pm}, b^{ \pm}, c_{ \pm}, d_{ \pm}, m_{ \pm}, v$ introduced in [SS2] before Lemma 8.3. Moreover, we set

$$
\begin{aligned}
b & :=b_{+}+\left(Q_{+} q_{-}-q^{-2}\right) b_{-}, e:=\left(Q_{+} q_{+}+1\right) a_{+}-q^{-2} a_{-}, \\
e^{+} & :=\left(Q_{+} q_{+}+q^{-2}+1-q^{2}\right) a_{+}+a_{-}, e^{-}:=b_{+}+\left(q^{-4}-q^{-2} Q_{+} q_{-}\right) b_{-} .
\end{aligned}
$$

Because im $S_{ \pm} \subseteq \mathscr{R}$ by Lemma 5.1, Lemma 8.3 in [SS2] and its proof remain valid verbation in the present more general situation. This is essential for most of the subsequent computations. 


\section{Lemma 5.2.}

(i) $\left(u_{ \pm}\right)_{m}^{n}=Q_{+}^{-1}\left(q^{\mp 1} u_{m}^{n} \pm K_{m m^{\prime}}^{n n^{\prime}} u_{n^{\prime}}^{m^{\prime}}\right)$ if $n^{\prime}>m, n \neq m$.

$\left(u_{ \pm}\right)_{m}^{n}=Q_{+}^{-1}\left(q^{ \pm 1} u_{m}^{n} \pm K_{m m^{\prime}}^{n n^{\prime}} u_{n^{\prime}}^{m^{\prime}}\right)$ if $n^{\prime}<m, n \neq m$.

(ii) $u_{m}^{n} u_{n^{\prime}}^{n^{\prime}} \equiv q Q_{+}^{-2} e^{+} u_{m}^{n}$ if $\left(\delta_{+}, \delta_{-}\right)=(0,1), n^{\prime}>m, n \neq m$ and $n \neq n^{\prime}$.

$u_{m}^{n} u_{n^{\prime}}^{n^{\prime}} \equiv q Q_{+}^{-2} e^{-} u_{m}^{n}$ if $\left(\delta_{+}, \delta_{-}\right)=(1,0), n^{\prime}>m, n \neq m$ and $n \neq n^{\prime}$.

(iii) $u_{n}^{1} u_{1}^{1^{\prime}} \equiv q Q_{+}^{-2} b u_{n}^{1^{\prime}}$ if $\left(\delta_{+}, \delta_{-}\right)=(1,0)$ and $1<n<1^{\prime}$.

(iv) $a_{+}=a_{-}=0$ if $\left(\delta_{+}, \delta_{-}\right)=(0,1)$.

(v) $q Q_{+}^{-3} e^{-} b_{+}=1$ and $b=0$ if $\left(\delta_{+}, \delta_{-}\right)=(1,0)$.

(vi) $b_{+}= \pm Q_{+}$and $b_{-}= \pm Q_{+}\left(q^{-1} z+1\right)\left(q^{-3} z+q^{2}\right)^{-1}$ if $\mathscr{A} \neq S p_{q}(4)$ and $\left(\delta_{+}, \delta_{-}\right)=(1,0)$

(vii) $\tilde{U} u_{j}^{l} \equiv(\psi-\mathfrak{s}) \tilde{u}_{j}^{i}+\delta_{i j} \mathfrak{s}^{-1}(\mu-\psi) \tilde{U}$ and $(\psi-\mathfrak{s})(\mu-\psi-\mathfrak{s})=0$ if $\left(\delta_{+}, \delta_{-}\right)=$ $(1,0)$.

Proof. (i) Both formulas are easily derived from the definitions of $\left(u_{+}\right)_{m}^{n}$ and $\left(u_{-}\right)_{m}^{n}$. (ii) We only carry out the proof of the first assertion. Since $\left(\delta_{+}, \delta_{-}\right)=(0,1)$, we have $\left(u_{+}\right)_{m}^{n} \equiv u_{m}^{n}-\delta_{n m} \mathfrak{s}^{-1} U$. Using this and the definitions of $F_{ \pm}, P_{ \pm}$and $a_{ \pm}$ (cf. [SS2]), we obtain

$$
\begin{aligned}
F_{ \pm}\left(u_{m}^{n} u_{n^{\prime}}^{n^{\prime}}\right) & =\sum_{i, j, k} \alpha_{ \pm}\left(P_{ \pm}\right)_{i j}^{n n^{\prime}}\left(f_{ \pm}\right)_{k}^{j}\left(P_{ \pm}\right)_{m n^{\prime}}^{i k} \\
& \equiv \alpha_{ \pm} \varphi^{ \pm}\left(\left(P_{ \pm}\right)_{m m^{\prime}}^{n n^{\prime}}\left(u_{+}\right)_{n^{\prime}}^{m^{\prime}}\left(P_{ \pm}\right)_{m n^{\prime}}^{m n^{\prime}}+\left(P_{ \pm}\right)_{n^{\prime} n}^{n n^{\prime}}\left(u_{+}\right)_{m}^{n}\left(P_{ \pm}\right)_{m n^{\prime}}^{n^{\prime} m}\right) \\
& =Q_{+}^{-2}\left(\left(q_{ \pm} \mp Q\right) K_{m m^{\prime}}^{n n^{\prime}} K_{n^{\prime} n}^{m^{\prime} m} q u_{m}^{n} a_{ \pm} q^{ \pm 1}+\left( \pm q^{-1}+q_{ \pm}\right)( \pm 1) a_{ \pm} u_{m}^{n}\right) \\
& =Q_{+}^{-2}\left(q q^{ \pm 1}\left(q_{ \pm} \mp Q\right) \pm\left( \pm q^{-1}+q_{ \pm}\right)\right) a_{ \pm} u_{m}^{n} .
\end{aligned}
$$

Since $\delta_{-}=1, \psi^{ \pm}=0$ and hence $\left(g_{ \pm}\right)_{j}^{i} \equiv 0$ Moreover, $\left(P_{ \pm}\right)_{m n^{\prime}}^{n n^{\prime}}=0$. Putting the preceding facts into formula (8.9) in [SS2] for the element $u_{m}^{n} u_{n^{\prime}}^{n^{\prime}}$, we get $u_{m}^{n} u_{n^{\prime}}^{n^{\prime}} \equiv$ $q Q_{+}^{-2} e^{+} u_{m}^{n}$.

(iii) From Lemma 8.3, (v.2), in [SS2], $u_{n}^{1} u_{1}^{1^{\prime}}=q u_{1}^{1^{\prime}} u_{n}^{1} \equiv q Q_{+}^{-3}\left(d_{-} u_{n}^{1^{\prime}}+K_{n n^{\prime}}^{1^{\prime} 1} c_{+} u_{1}^{n^{\prime}}\right)$. Since $\left(\delta_{+}, \delta_{-}\right)=(1,0)$, we have $\left(u_{+}\right)_{j}^{i} \equiv 0$, so that $u_{1}^{n^{\prime}} \equiv-q K_{11^{\prime}}^{n^{\prime} n} u_{n}^{1^{\prime}}$. Moreover, $\varphi^{ \pm}=0$. Inserting these facts into the above expression for $u_{n}^{1} u_{1}^{1^{\prime}}$, we compute that $u_{n}^{1} u_{1}^{1^{\prime}} \equiv q Q_{+}^{-2} b u_{n}^{1^{\prime}}$.

(iv) We first show that $a_{+} e^{+}=0$. From Lemma 8.3, (iii.2), in [SS2] and the first assertion of (ii), we obtain

$$
u_{1}^{1} u_{1}^{1^{\prime}} u_{2}^{1}=q^{2} u_{1}^{1^{\prime}} u_{1}^{1} u_{2}^{1} \equiv q^{2} v a_{+} u_{1}^{1^{\prime}} u_{2}^{1}=q v a_{+} u_{2}^{1} u_{1}^{1^{\prime}} \equiv q^{2} v Q_{+}^{-2} a_{+} e^{+} u_{2}^{1^{\prime}}
$$

On the other hand, since $\left(u_{+}\right)_{2}^{1} \equiv u_{2}^{1}$ and $\left(u_{-}\right)_{2}^{1} \equiv 0$ by $\left(\delta_{+}, \delta_{-}\right)=(0,1)$, Lemma 8.3, (iii.1), in [SS2] and (ii) yield

$$
u_{1}^{1} u_{1}^{1^{\prime}} u_{2}^{1}=u_{2}^{1} u_{1}^{1} u_{1}^{1^{\prime}} \equiv Q_{+}^{-1} a_{+} u_{2}^{1} u_{1}^{1^{\prime}} \equiv Q_{+}^{-3} q a_{+} e^{+} u_{2}^{1^{\prime}} .
$$

Since $u_{2}^{1^{\prime}} \notin \mathscr{R}$ again by $\left(\delta_{+}, \delta_{-}\right)=(0,1)$, the above coefficients of $u_{2}^{1^{\prime}}$ coincide so that $q Q_{+} v a_{+} e^{+}=a_{+} e^{+}$. Since $q Q_{+} v=1+q q_{+} \neq 1, a_{+} e^{+}=0$.

From $\left(\delta_{+}, \delta_{-}\right)=(0,1)$ it follows easily that $u_{2^{\prime}}^{1} u_{1^{\prime}}^{2^{\prime}} \equiv q^{-1} K_{2^{\prime}}^{11^{\prime}} u_{1^{\prime}}^{2} u_{1^{\prime}}^{2^{\prime}}$. By the assertions (iv.1) and (vi.1) of Lemma 8.3 in [SS2], we have $u_{2^{\prime}}^{1} u_{1^{\prime}}^{2^{\prime}} \equiv Q_{+}^{-2} a^{+} u_{1^{\prime}}^{1}$ and 
$q^{-1} K_{2^{\prime}}^{11^{\prime}} u_{1^{\prime}}^{2} u_{1^{\prime}}^{2^{\prime}} \equiv q^{-1} Q_{+}^{-1} q_{+} a_{+} u_{1^{\prime}}^{1}$, where we used that $\left(u_{-}\right)_{1^{\prime}}^{1}=0$. Since $\left(\delta_{+}, \delta_{-}\right)=$ $(0,1), u_{1^{\prime}}^{1}$ does not belong to $\mathscr{R}$. Comparing the coefficients of $u_{1^{\prime}}^{1}$, we get $Q_{+}^{-1} a^{+}=$ $q^{-1} q_{+} a_{+}$. From the latter and the equation $a_{+} e^{+}=0$ we compute immediately that $a_{+}=a_{-}=0$.

(v) Similarly to formula $(8.10)$ in [SS2] we can prove the identity

$$
C_{1}^{1^{\prime}} u_{2}^{1} \equiv C_{2^{\prime}}^{2} u_{2}^{1} u_{1^{\prime}}^{2} u_{1}^{2^{\prime}}+C_{1}^{1^{\prime}} u_{2}^{1} u_{1^{\prime}}^{1^{\prime}} u_{1}^{1} \text {. }
$$

We have $\left(u_{-}\right)_{1^{\prime}}^{1}=0$. Since $\delta_{+}=1,\left(u_{+}\right)_{1^{\prime}}^{1} \equiv 0$. Therefore, by Lemma 8.3 , (iv), in [SS2], $u_{2}^{1} u_{1^{\prime}}^{2} u_{1}^{2^{\prime}} \equiv 0$. From the second assertion of (ii) and Lemma 8.3, (iii.1), in [SS2], we get $u_{2}^{1} u_{1^{\prime}}^{\prime} u_{1}^{1} \equiv q Q_{+}^{-2} e^{-} u_{2}^{1} u_{1}^{1} \equiv q Q_{+}^{-3} e^{-} b_{+} u_{2}^{1}$. Here we also used the fact that $\left(u_{-}\right)_{2}^{1} \equiv u_{2}^{1}$ and $\left(u_{+}\right)_{2}^{1} \equiv 0$. Comparing the coefficient of $u_{2}^{1} \notin \mathscr{R}$, we obtain $1=q Q_{+}^{-3} e^{-} b_{+}$. Since $\left(\delta_{+}, \delta_{-}\right)=(1,0)$, we have $u_{1}^{1^{\prime}} \equiv 0$. Hence, by (ii), $0 \equiv$ $u_{1}^{1^{\prime}} u_{2}^{1}=q^{-1} u_{2}^{1} u_{1}^{1^{\prime}} \equiv Q_{+}^{-2} b u_{2}^{1^{\prime}}$. Because $u_{2}^{1^{\prime}} \notin \mathscr{R}$, this gives $b=0$.

(vi) is easily computed from (v) and the definitions of $b_{ \pm}, b$ and $e^{-}$. Note that $q^{-3} z+q^{2} \neq 0$, since $\mathscr{A} \neq S p_{q}(4)$ and hence $z \neq-q^{5}$.

(vii) Since $\delta_{+}=1$ and hence $\varphi^{ \pm}=0$, we have $u_{j}^{i} \equiv\left(u_{-}\right)_{j}^{i}+\delta_{i j} \mathfrak{s}^{-1} U$ and $\left(f_{ \pm}\right)_{j}^{i} \equiv$ 0 . Thus

$$
\begin{aligned}
\tilde{U} u_{j}^{i} & =\left(v_{+}\right)_{j}^{l}+\left(v_{-}\right)_{j}^{i}+\delta_{i j \mathfrak{s}}^{-1} \mathbb{1}-\mathfrak{s} u_{j}^{i} \\
& =\left(f_{+}\right)_{j}^{i}+\left(f_{-}\right)_{j}^{i}+\left(g_{+}\right)_{j}^{i}+\left(g_{-}\right)_{j}^{i}+\delta_{i j} \mathfrak{s}^{-1}\left(\mathbb{1}+V_{+}+V_{-}\right)-\mathfrak{s} u_{j}^{i} \\
& \equiv\left(\psi^{+}+\psi^{-}\right)\left(u_{-}\right)_{j}^{i}+\delta_{i j} \mathfrak{s}^{-1}\left(\mu^{+}+\mu^{-}\right) \tilde{U}-\mathfrak{s} \tilde{u}_{j}^{i} \\
& =(\psi-\mathfrak{s}) \tilde{u}_{j}^{i}+\delta_{i j \mathfrak{s}}{ }^{-1}(\mu-\psi) \tilde{U} .
\end{aligned}
$$

Applying the first assertion to the identity $C_{1^{\prime}}^{1} \tilde{U}=\sum_{i} C_{i^{\prime}}^{i} \tilde{U} u_{1}^{i} u_{1^{\prime}}^{i^{\prime}}$ and using the fact that $\tilde{U}^{2}=V_{+}+V_{-}-2 \mathfrak{s} \tilde{U}+\left(1-\mathfrak{s}^{2}\right) \mathbb{1}=(\mu-2 \mathfrak{s}) \tilde{U}$, we obtain

$$
C_{1^{\prime}}^{1} \tilde{U} \equiv C_{1^{\prime} \mathfrak{s}}^{1}{ }^{-1}(\psi-\mathfrak{s})(\mu-\psi-\mathfrak{s}){\tilde{u}_{1^{\prime}}^{\prime}}^{\prime}+C_{1^{\prime} \mathfrak{s}^{-2}}^{1}(\mu-\psi)^{2} \tilde{U}
$$

Since $\tilde{u}_{1^{\prime}}^{1^{\prime}}$ and $\tilde{U}$ are linearly independent modulo $\mathscr{R}$, we conclude that $(\psi-\mathfrak{s})(\mu-$ $\psi-\mathfrak{s})=0$.

Now we continue the discussion of the remaining cases for the numbers $\delta_{0}, \delta_{+}, \delta_{-} \in\{0,1\}$. First we assume that $\delta_{+}=0$ and $\delta_{-}=1$. Then $u_{1}^{1^{\prime}} \equiv\left(u_{+}\right)_{1}^{1^{\prime}} \notin \mathscr{R}$. Therefore, the proof of assertion (i) of Lemma 8.4 in [SS2] is still valid in this case and shows that $Q_{+} q_{+} a_{+} d_{-}+a^{+} c_{+}=Q_{+}^{5}-Q_{+}\left(a_{+}+a_{-}\right)^{2}$. By Lemma 5.2, (iv), $a_{+}=a_{-}=0$, and hence $a^{+}=0$. Thus we get $Q_{+}=0$, which is impossible since $q$ is not a root of unity.

In the rest of this we consider the case $\delta_{+}=1, \delta_{-}=0$.

Next we suppose that $N \geqq 5$. From Lemma 8.3, (i), in [SS2], $u_{2}^{1} u_{3}^{2} u_{2}^{3}=$ $u_{2}^{1} u_{2}^{3} u_{3}^{2} \equiv 0$. Applying both assertions of Lemma 8.3, (iv), in [SS2], we obtain $0 \equiv u_{2}^{1} u_{3}^{2} u_{2}^{3}=Q_{+}^{-2} b^{+} u_{3}^{1} u_{2}^{3} \equiv Q_{+}^{-4} b^{+} b^{-} u_{2}^{1}$, where we also used the relations $\left(u_{+}\right)_{3}^{1} \equiv$ $0,\left(u_{-}\right)_{3}^{1} \equiv u_{3}^{1},\left(u_{+}\right)_{2}^{1} \equiv 0$ and $\left(u_{-}\right)_{2}^{1} \equiv u_{2}^{1}$. (Note that for $N=4$ we have $3=2^{\prime}$, so that Lemma 8.3, (iv), does not apply to the terms $u_{3}^{1} u_{2}^{3}$ in this case.) Since $u_{2}^{1} \notin \mathscr{R}$, we conclude that $b^{+} b^{-}=0$. On the other hand, Lemma 5.2, (vi), implies that $b^{+} b^{-}=-q^{-1} Q^{2} Q_{+}^{4} z\left(q^{-3} z+q^{2}\right)^{-2} \neq 0$, which is a contradiction. 
Now suppose that $\mathscr{A}=S p_{q}(4)$. In this case we have $\left(g_{-}\right)_{j}^{i}=0$ for all $i, j$ and hence $\psi^{-}=0$, cf. Lemma 4.1. Therefore, $b_{-}=0$. From Lemma 5.2, (v), $b=0$ and $q Q_{+}^{-3} e^{-} b_{+}=1$. But $b_{-}=0$ and $b=0$ imply $b_{+}=0$, which leads to a contradiction.

It only remains to treat the quantum groups $O_{q}(4)$ and $O_{q}(3)$. Let us suppose that $\mathscr{A}=O_{q}(4)$ or $\mathscr{A}=O_{q}(3)$. Recall that $\left(\delta_{+}, \delta_{-}\right)=(1,0)$ as assumed above.

From the two solutions $b_{+}= \pm Q_{+}, b_{-}= \pm Q_{+}\left(q^{-1} z+1\right)\left(q^{-3} z+q^{2}\right)^{-1}$ by Lemma 5.2, (vi), we obtain the two solutions $\psi_{1}^{ \pm}$and $\psi_{2}^{ \pm}$, where $\psi_{1}^{+}=-\psi_{2}^{+}:=$ $Q^{-1} Q_{+}^{-1}\left(z+q^{-1}\right)\left(q^{2}-q^{-1} z^{-1}\right)$ and $\psi_{1}^{-}=-\psi_{2}^{-}:=Q^{-1} Q_{+}^{-1}(z-q)\left(1+q^{-1} z^{-1}\right)$. We set $\psi_{k}:=\psi_{k}^{+}+\psi_{k}^{-}, k=1,2$. We compute $\psi_{1}-\mathfrak{s}=Q_{+}(1-q)\left(q^{-2}-q\right), \psi_{2}-$ $\mathfrak{s}=Q_{+}(1+q)\left(q^{-2}+q\right)$ for $N=4$ and $\psi_{1}-\mathfrak{s}=(1-q)\left(q^{-2}-q\right), \psi_{2}-\mathfrak{s}=-Q_{+}$ $\left(Q_{+}+1\right)$ for $N=3$. Therefore, since $q$ is not a root of unity, we have $\psi-\mathfrak{s} \neq 0$ in all possible cases.

In order to determine $m_{+}$and so $\mu^{+}$, we begin with the identity $u_{1}^{1} u_{1}^{1} u_{2}^{1}=$ $q^{2} u_{2}^{1} u_{1}^{1} u_{1}^{1}$. From Lemma 8.3, (vii) and (iii), in [SS2] and the relation $\tilde{U} u_{2}^{1} \equiv(\psi-$ s ) $u_{2}^{1}$ by Lemma 5.2, (vii), above, we get

$$
u_{1}^{1} u_{1}^{1} u_{2}^{1} \equiv\left(b_{+}\left(q Q_{+}^{-1} b_{+}-\mathfrak{s}^{-1} \psi\right)+m_{+}(\psi-\mathfrak{s})+1\right) u_{2}^{1} .
$$

Here we also used $\left(u_{+}\right)_{j}^{i} \equiv 0$ and $u_{j}^{l} \equiv\left(u_{-}\right)_{j}^{i}+\delta_{i j} \mathfrak{s}^{-1} U$ by $\left(\delta_{+}, \delta_{-}\right)=(1,0)$. Applying Lemma 8.3 , (iii), in [SS2] twice, we obtain $u_{2}^{1} u_{1}^{1} u_{1}^{1} \equiv Q_{+}^{-2} b_{+}^{2} u_{2}^{1}$. Comparing the coefficients of $u_{2}^{1} \notin \mathscr{R}$ and using the facts that $\psi-\mathfrak{s} \neq 0$ and $b_{+}^{2}=Q_{+}^{2}$ by Lemma 5.2 , (vi), we find that $m_{+}=\left(b_{+} \mathfrak{s}^{-1} \psi-2\right)(\psi-\mathfrak{s})^{-1}$. Setting in case $\mathscr{A}=O_{q}(3)$ the above solutions $\psi_{k}^{ \pm}, k=1,2$, into the latter formula, we obtain $\mu^{+}=\mu_{k}^{+}$.

Now we specialize to $\mathscr{A}=O_{q}(4)$. By Lemma 8.3 , (i), in [SS2], we have $u_{2}^{1} u_{2}^{1} \equiv$ 0 . Applying first assertion (viii) and then assertion (iii) of Lemma 8.3 in [SS2] to the term $u_{2}^{1} u_{1}^{2} u_{2}^{1}$, we conclude from $0 \equiv u_{2}^{1} u_{2}^{1} u_{1}^{2}=u_{2}^{1} u_{1}^{2} u_{2}^{1}$ that

$$
\begin{aligned}
0 \equiv & \left\{Q_{+}^{-1}\left(b^{+}\left(u_{2}^{2}-\mathfrak{s}^{-1} U\right)+b^{-}\left(u_{1}^{1}-\mathfrak{s}^{-1} U\right)+\left(m_{+}-m_{-}\right) \tilde{U}\right\} u_{2}^{1}\right. \\
\equiv & \left\{Q_{+}^{-2} b_{+}\left(q^{-1} b^{+}+q b^{-}\right)-Q_{+}^{-1} \mathfrak{s}^{-1} \psi\left(b^{+}+b^{-}\right)\right. \\
& \left.+\left(m_{+}-m_{-}\right)(\psi-\mathfrak{s})\right\} u_{2}^{1} .
\end{aligned}
$$

Since $u_{2}^{1} \notin \mathscr{R}$, the coefficient of $u_{2}^{1}$ vanishes. Combined with the above expression for $m_{+}$, this yields $m_{-}=Q\left(q^{-2} z-q\right)\left(q^{-3} z+q^{2}\right)^{-1}(\psi-\mathfrak{s})^{-1}$. Inserting the two solutions $\psi_{k}^{ \pm}$into the formulas for $m_{ \pm}$, we compute $\mu_{1}=\mu_{1}^{+}+\mu_{1}^{-}=Q_{+}^{-1}\left(q^{2}+q+\right.$ 1) $\left(q+q^{-2}\right)^{2}, \mu_{2}=\mu_{2}^{+}+\mu_{2}^{-}=\left(Q_{+}^{-1}-1\right)\left(q^{3}-1\right)^{2} q^{-3}$. Moreover, $\psi_{1}+\mathfrak{s}=Q_{+}(1+$ $q)\left(q+q^{-2}\right)$ and $\psi_{2}+\mathfrak{s}=Q_{+}(1-q)\left(q-q^{-2}\right)$. Using once more that $q$ is not root of unity, we verify from these formulas that $\mu-\psi-\mathfrak{s} \neq 0$ for both solutions. Since $\psi-\mathfrak{s} \neq 0$ as observed above, $(\psi-\mathfrak{s})(\mu-\psi-\mathfrak{s}) \neq 0$, which contradicts Lemma 5.2 , (vii).

Putting the preceding together, we have shown that for $N \geqq 4$ there are no other ad-invariant right ideals $\mathscr{R}$ of ker $\varepsilon$ satisfying codim $\mathscr{R} \geqq 2$ and ker $\varepsilon=\mathscr{R}+\tilde{\mathscr{A}}_{1}$ than the right ideals $\mathscr{R}_{+}$and $\mathscr{R}_{-}$defined in [SS2], Sect. 6. Therefore, by the Theorems 6.1 and 6.2 of our previous paper [SS2], the proof of Theorem 4.1 is complete, and the assertions concerning the right ideals $\mathscr{R}_{ \pm}$and the calculi $\left(\Gamma_{ \pm}, \mathrm{d}\right)$ in Theorem 4.2 are proven. 
From now on we assume that $\mathscr{A}=O_{q}(3)$. Our aim is to prove the remaining parts of Theorem 4.2. First we note that $\delta_{0}=0$, because otherwise $\tilde{U} \in \mathscr{R}$ and hence $0 \equiv \tilde{U} u_{2}^{1} \equiv(\psi-\mathfrak{s}) u_{2}^{1}$ by Lemma 5.2, (vii). Since $u_{2}^{1} \notin \mathscr{R}$ and $\psi-\mathfrak{s} \neq 0$, this is impossible. From Lemma 5.2, (vii), and $\psi-\mathfrak{s} \neq 0$ we obtain $\mu=\psi+\mathfrak{s}$. Putting the solutions $\psi_{k}^{ \pm}$and $\mu_{k}^{+}, k=1,2$, derived above into this equation, we compute $\mu^{-}=\mu_{k}^{-}$. (It might be of interest to search for the difference between the quantum groups $O_{q}(4)$ and $O_{q}(3)$ in the paragraph before last. The relation (5.4) still holds for $O_{q}(3)$, since $u_{1^{\prime}}^{1} \equiv\left(u_{+}\right)_{1^{\prime}}^{1} \equiv 0$ by $\delta_{+}=1$ and hence $u_{2}^{1} u_{2}^{1} \equiv 0$ by Lemma 8.3 , (vi.2), in [SS2]. But, since $2=2^{\prime}$ for $O_{q}(3)$, Lemma 8.3, (iii), in [SS2] does not apply to the term $u_{2}^{2} u_{2}^{1}$ in (5.4). A direct computation based on formula (8.9) in [SS2] yields that $u_{2}^{2} u_{2}^{1} \equiv Q_{+}^{-1} b_{+} u_{2}^{1}$ for $O_{q}(3)$, while $u_{2}^{2} u_{2}^{1} \equiv q^{-1} Q_{1}^{-1} b_{+} u_{2}^{1}$ for $O_{q}(4)$. The reason for this difference is that $\left(P_{ \pm}\right)_{22}^{22}=q^{\mp 1} \pm 1+q_{ \pm}$for $O_{q}(3)$, while $\left(P_{+}\right)_{22}^{22}=1$ and $\left(P_{-}\right)_{22}^{22}=0$ for $O_{q}(4)$. Therefore, in the corresponding relation (5.5) for $O_{q}(3)$ the factor $q^{-1}$ in the first interior bracket is missing and hence no contradiction appears in the case of $O_{q}(3)$.)

Thus, by the preceding computations and by Lemma 5.1, we have shown that $\mathscr{R}_{12}=\mathscr{B}_{1}$ or $\mathscr{R}_{12}=\mathscr{B}_{2}$. Hence we have $\mathscr{R}=\mathscr{R}_{1}$ or $\mathscr{R}=\mathscr{R}_{2}$ by Lemma 1.1 in [SS2]. The right ideals $\mathscr{R}_{1}$ and $\mathscr{R}_{2}$ are ad-invariant by Lemma 1.7 in [W], since the generating subspaces $\mathscr{B}_{1}$ and $\mathscr{B}_{2}$ are ad-invariant. From the definitions of $\mathscr{R}_{k}$ it is clear that ker $\varepsilon=\mathscr{R}_{k}+\mathscr{A}_{1}$ and codim $\mathscr{R}_{k} \leqq 4$. Later in this proof we will conclude that codim $\mathscr{R}_{k}=4$ for $k=1,2$. In the next lemma we take this already for granted.

Lemma 5.3. If codim $\mathscr{R}_{1} \geqq 2$ and codim $\mathscr{R}_{2} \geqq 2$, then we have $\tilde{\mathscr{D}} \in \mathscr{R}_{1}$ and $\tilde{\mathscr{D}}+2\left(\psi_{2}-\mathfrak{s}\right)^{-1} \tilde{U} \in \mathscr{R}_{2}$.

Proof. Let $k \in\{1,2\}$. Since ker $\varepsilon=\mathscr{R}_{k}+\tilde{\mathscr{A}_{1}}$ by definition, there is an element $a_{k} \in \tilde{\mathscr{A}}_{1}$ such that $\tilde{\mathscr{D}}-a_{k} \in \mathscr{R}_{k}$. Since the quantum determinant is group-like [H] and hence ad-invariant, it follows that $a_{k}=\alpha_{k} \tilde{U}$ for some $\alpha_{k} \in \mathbb{C}$, so that $\tilde{\mathscr{D}} \equiv \alpha_{k} \tilde{U}$.

Now we determine the complex number $\alpha_{k}$. Recall that the quantum determinant $\mathscr{D}$ is central in $O_{q}(3)$. Therefore, by (4.1), we have

$$
\mathscr{D} u_{2}^{1}=u_{2}^{1} \mathscr{D}=u_{2}^{1} u_{1}^{1} u_{2}^{2} u_{3}^{3}-(1+q) u_{2}^{1} u_{1}^{1} u_{3}^{2} u_{2}^{3}+\left(q+q^{2}\right) u_{2}^{1} u_{2}^{1} u_{3}^{2} u_{1}^{3}-q^{2} u_{2}^{1} u_{3}^{1} u_{2}^{2} u_{1}^{3}
$$

To compute the right-hand side of (5.6) modulo $\mathscr{R}_{k}$, we apply once again Lemma 8.3 in [SS2]. All references in this paragraph will refer to assertions of that lemma. By (i), $u_{2}^{1} u_{3}^{1} \equiv 0$, so that $u_{2}^{1} u_{3}^{1} u_{2}^{2} u_{1}^{3} \equiv 0$. From $u_{1^{\prime}}^{1}=\left(u_{+}\right)_{1^{\prime}}^{1} \in \mathscr{R}_{k}$ and (vi.2) we conclude that $u_{2}^{1} u_{2}^{1} \equiv 0$. Hence $u_{2}^{1} u_{2}^{1} u_{3}^{2} u_{1}^{3} \equiv 0$. Since $b_{+}=(-1)^{k+1} Q_{+}$as computed above and $\left(u_{+}\right)_{2}^{1} \in \mathscr{R}_{k}$, we have $u_{2}^{1} u_{1}^{1} \equiv u_{2}^{1} u_{2}^{2} \equiv(-1)^{k+1} u_{2}^{1}$. From $u_{1^{\prime}}^{1} \in \mathscr{R}_{k}$ and (iii.2) we get $u_{2}^{1} u_{3}^{2} \equiv 0$. Thus $u_{2}^{1} u_{1}^{1} u_{3}^{2} u_{2}^{3} \equiv 0$. Therefore, by (5.6) and the preceding, $\mathscr{D} u_{2}^{1} \equiv u_{2}^{1} u_{1}^{1} u_{2}^{2} u_{3}^{3} \equiv(-1)^{k+1} u_{2}^{1} u_{2}^{2} u_{3}^{3} \equiv u_{2}^{1} u_{3}^{3}$.

Further, we have $u_{2}^{1} u_{3}^{3}=u_{2}^{1} u_{1^{\prime}}^{1^{\prime}} \equiv q Q_{+}^{-2} e^{-} u_{2}^{1}$ by Lemma 5.2, (ii), and $1=$ $q Q_{+}^{-3} b_{+} e^{-}=(-1)^{k+1} q Q_{+}^{-1} e^{-}$by Lemma 5.2 , (v), so that $\tilde{\mathscr{D}} u_{2}^{1} \equiv(-1)^{k+1} u_{2}^{1}$. Thus we get $\tilde{\mathscr{D}} u_{2}^{1}=(\mathscr{D}-\mathbb{1}) u_{2}^{1} \equiv\left((-1)^{k+1}-1\right) u_{2}^{1} \equiv \alpha_{k} \tilde{U} u_{2}^{1} \equiv \alpha_{k}(\psi-\mathfrak{s}) u_{2}^{1}$, where the last relation follows from (5.3). Since codim $\mathscr{R}_{k} \geqq 2$ by the above assumption, we have $u_{2}^{1} \notin \mathscr{R}_{k}$. Hence we conclude that $(-1)^{k+1}-1=\alpha_{k}(\psi-\mathfrak{s})$, which yields $\alpha_{1}=0$ and $\alpha_{2}=-2(\psi-\mathfrak{s})^{-1}$. 
Next we turn to the differential calculi $\left(\Gamma_{1}, \mathrm{~d}\right)$ and $\left(\Gamma_{2}, \mathrm{~d}\right)$. First we show $\Gamma_{k}=$ $\left\langle a \mathrm{~d} u_{s}^{r}: a \in \mathscr{A}, r, s=1,2,3\right\rangle$ for $k=1,2$. Throughout this proof let $\left(\hat{R}_{n m}^{i j}\right)$ denote the matrix $\hat{R}$ for the quantum group $S L_{p}(2)$. Recall that $(\tilde{\Gamma}, \mathrm{d})$ is the calculus $\left(\Gamma_{+, z}, \mathrm{~d}\right)$ with $z=p^{-1}$ for $S L_{p}(2)$ defined in Sect. 2. From the corresponding formulas therein we compute

$$
\begin{aligned}
\kappa\left(t_{k}^{i} t_{l}^{j}\right) \mathrm{d}\left(t_{n}^{k} t_{m}^{l}\right) & =\kappa\left(t_{k}^{i} t_{l}^{j}\right) \eta t_{n}^{k} t_{m}^{l}-\delta_{i n} \delta_{j m} \eta=p^{-2} p^{-2 a} \hat{R}_{b c}^{a i} \hat{R}_{a n}^{b x} \hat{R}_{y r}^{c j} \hat{R}_{x m}^{y s} \eta_{r s}-\delta_{i n} \delta_{j m} \eta \\
& =p^{-3}\left(p-p^{-1}\right)\left(\hat{R}_{y r}^{i j} \hat{R}_{n m}^{y s} \eta_{r s}+\delta_{i n} \eta_{j m}\right)+\left(p^{-2}-1\right) \delta_{i n} \delta_{j m} \eta
\end{aligned}
$$

Here we used the equation $p^{-2 a} \hat{R}_{b c}^{a i} \hat{R}_{a n}^{b x}=\left(\operatorname{tr}_{12} \dot{R}_{12} R_{23}\right)_{\text {in }}^{c x}=p^{-1}\left(p-p^{-1}\right) \delta_{c i} \delta_{x n}+$ $p^{-2 c} \delta_{c x} \delta_{\text {in }}$ from Lemma 3.4, (iii), in [SS2]. The right-hand side of (5.7) is equal to $p^{-3}\left(p-p^{-1}\right) \eta_{j m}$ if $i=j \neq n=m, p^{-3}\left(p-p^{-1}\right) 2 \eta_{i i}+\left(p^{-2}-1\right) \eta$ if $i=$ $n>j=m$, and $p^{-3}\left(p-p^{-1}\right)\left(p^{2}+1\right) \eta_{i i}+\left(p^{-2}-1\right) \eta$ if $i=j=m=n$. Since $t_{n}^{k} t_{m}^{l} \in \pi(\mathscr{A})$, we conclude that for the pair $\left(\Gamma_{1}, \mathrm{~d}\right)$ all forms $\eta_{i j}, i, j=1,2$, belong to the linear span $\left\langle a \mathrm{~d} u_{s}^{r}: a \in \mathscr{A}, r, s=1,2,3\right\rangle$. Hence the latter coincides with $\Gamma_{1}$. Recall that we have $\eta_{i j} u_{s}^{r}=(-1)^{k+1} u_{l}^{r} g_{n m}^{i j}\left(u_{s}^{l}\right) \eta_{n m}$ for the bimodule $\Gamma_{k}, k=1,2$. Therefore, a slight modification of the preceding reasoning shows that $\Gamma_{2}=\left\langle a \mathrm{~d} u_{s}^{r}: a \in\right.$ $\mathscr{A}, r, s=1,2,3\rangle$.

In particular, we have $\Gamma_{1}=\langle a \mathrm{~d} b: a, b \in \mathscr{A}\rangle$, so that $\left(\Gamma_{1}, \mathrm{~d}\right)$ is indeed a differential calculus over $\mathscr{A}=O_{q}(3)$. Since $\pi: O_{q}(3) \rightarrow S L_{p}(2)$ is a Hopf algebra morphism and the $4 D_{+}$-calculus $(\tilde{\Gamma}, \mathrm{d})$ over $S L_{p}(2)$ is bicovariant, the calculus $\left(\Gamma_{1}, \mathrm{~d}\right)$ over $O_{q}(3)$ is also bicovariant. The easiest way to see this is to use the map $\Psi: \Gamma_{1} \rightarrow \Gamma_{+, z}$ defined in Sect. 4 and to check Definitions 1.2 and 1.3 in [W] (which are equivalent to our definition of bicovariance given in the introduction). Since $\Gamma_{1}=(w, g)$ is a bicovariant bimodule, it follows immediately from Lemma 1.3 in [SS2] and the definition of the algebra representation $h$ that $\Gamma_{2}=(w, h)$ is again a bicovariant bimodule. Hence $\left(\Gamma_{2}, \mathrm{~d}\right)$ is also a bicovariant differential calculus over $\mathscr{A}=O_{q}(3)$.

By a direct computation based on formula (4.1) we verify that $\pi(\mathscr{D})=\mathbb{1}$. From this it follows easily that $\eta \mathscr{D}=\mathscr{D} \eta$ for the bimodule $\Gamma_{1}$. Since $\mathscr{D}$ is a linear combination of cubic monomials in the matrix entries $u_{j}^{i}$, it follows at once from the definition of the bimodule $\Gamma_{2}$ that $\eta \mathscr{D}=-\mathscr{D} \eta$ in $\Gamma_{2}$. Thus we have $\mathrm{d} \mathscr{D}=-2 \mathscr{D} \eta \neq 0$ for the calculus $\left(\Gamma_{2}, \mathrm{~d}\right)$. In particular, we see that the two calculi $\left(\Gamma_{1}, \mathrm{~d}\right)$ and $\left(\Gamma_{2}, \mathrm{~d}\right)$ are not isomorphic.

Let $\mathscr{R}_{k}^{\prime}, k=1,2$, be the ad-invariant right ideal of ker $\varepsilon$ which is associated with the calculus $\left(\Gamma_{k}, \mathrm{~d}\right)$, cf. [W]. Since $\operatorname{dim}\left(\Gamma_{k}, \mathrm{~d}\right)=4$ by construction and $\Gamma_{k}=$ $\left\langle a \mathrm{~d} u_{j}^{i}: a \in \mathscr{A}, i, j=1,2,3\right\rangle$ as just shown, we have codim $\mathscr{R}_{k}^{\prime}=4$ and ker $\varepsilon=$ $\mathscr{R}_{k}^{\prime} \tilde{\mathscr{A}}_{1}$ by Lemma 1.5 in [SS2]. Therefore, by the above proof, each $\mathscr{R}_{k}^{\prime}$ has to be one of right ideals $\mathscr{R}_{1}$ or $\mathscr{R}_{2}$ of ker $\varepsilon$. Further, we know that $\mathscr{R}_{1}^{\prime} \neq \mathscr{R}_{2}^{\prime}$, because the calculi $\left(\Gamma_{1}, \mathrm{~d}\right)$ and $\left(\Gamma_{2}, \mathrm{~d}\right)$ are not isomorphic. In particular, this implies that codim $\mathscr{R}_{1}=$ codim $\mathscr{R}_{2}=4$. Thus Lemma 5.3 applies, and hence we have $\tilde{\mathscr{D}} \in \mathscr{R}_{1}$ and $\tilde{\mathscr{D}} \notin \mathscr{R}_{2}$. Since $\mathrm{d} \mathscr{D}=0$ for $\left(\Gamma_{1}, \mathrm{~d}\right)$ and $\mathrm{d} \mathscr{D} \neq 0$ for $\left(\Gamma_{2}, \mathrm{~d}\right)$, we conclude that $\mathscr{R}_{1}^{\prime}=\mathscr{R}_{1}$ and $\mathscr{R}_{2}^{\prime}=\mathscr{R}_{2}$.

This completes the proof of Theorem 4.2. 


\section{References}

[BM] Brzeziński, T., Majid,S.: Quantum group gauge theory on quantum spaces. Commun. Math. Phys. 157, 591-638 (1993)

[CSSW] Carow-Watamura, U., Schlieker, M., Watamura, S., Weich, W.: Bicovariant differential calculus on quantum groups $S U_{q}(N)$ and $S O_{q}(N)$. Commun. Math. Phys. 142, 605-641 (1991)

[C] Castellani, L.: Gauge theories of quantum groups. Phys. Lett. B292, 93-98 (1992)

[D] Drinfeld, V.G.: Quantum groups. In: Proceedings ICM 1986, pp. 798-820, Providence, RI : Am. Math. Soc., 1987

[DK] Dijkhuizen, M.S., Koornwinder, T.W.: Quantum homogeneous spaces, duality and quantum 2-spheres. Preprint, Amsterdam, 1993

[FRT] Faddeev, L.D., Reshetikhin, N.Yu., Takhtajan, L.A.: Quantization of Lie groups and Lie algebras. Algebra and Analysis 1, 178-206 (1987)

[Hm] Hammermesh, M.: Group theory and its application to physical problems. Reading, MA: Addison-Wesley, 1992

[H] Hayashi, T.: Quantum groups and quantum determinants. J. Algebra 152, 146-165 (1992)

[J] Jimbo, M.: A q-difference analogue of $U(g)$ and the Yang-Baxter equation. Lett. Math. Phys. 10, 63-65 (1985)

[Ju] Jurčo, B.: Differential calculus on quantized simple Lie groups. Lett. Math. Phys. 22, $177-186(1991)$

[L] Lusztig, G.: Quantum deformations of certain simple modules over enveloping algebras. Adv. Math. 70, 237-249 (1988)

[Mj] Majid, S.: Quasitringular Hopf algebras and Yang-Baxter equations. Int. J. Mod. Phys. A5, 1-91 (1990)

[M1] Maltsiniotis, G.: Calcul différential sur le groupe lineáire quantique. Preprint, ENS, Paris, 1990

[M1] Manin, Yu. I.: Quantum groups and non-commutative geometry. Publications du C.R.M. 1561, Univ. of Montreal, (1988)

[M2] Manin, Yu. I.: Notes on quantum groups and quantum de Rham complexes. Preprint, Max-Planck-Institut, Bonn, (1991)

[MH] Müller-Hoissen, F.: Differential calculi on the quantum group $G L_{p, q}(2)$. J. Phys. A. Math. Gen. 25, 1703-1734 (1992)

[S] Sudberry, A.: Canonical differential calculus on quantum linear groups and supergroups. Phys. Lett. B 284, 61-65 (1992)

[PW] Parshall, B., Wang, I.: Quantum linear groups. Memoirs Am. Math. Soc. 439, Providence, RI (1991)

[R] Rosso, M.: Finite dimensional representations of the quantum analog of the enveloping algebra of a complex simple Lie algebra. Commun. Math. Phys. 117, 581-593 (1988)

[SS1] Schmüdgen, K., Schüler, A.: Covariant differential calculi on quantum spaces and on quantum groups. C.R. Acad. Sci. Paris 316, 1155-1160 (1993)

[SS2] Schmüdgen, K., Schüler, A.: Classification of bicovariant calculi on quantum groups of type $A, B, C$ and $D$. Commun. Math. Phys. 167, 635-670 (1995)

[SWZ] Schupp, P., Watts, P., Zumino, B.: Differential Geometry on Linear Quantum Groups. Lett. Math. Phys. 25, 139-147 (1992)

[T] Takeuchi, M.: Matric bialgebras and quantum groups. Israel J. Math. 72, 232-251 (1990)

[W] Woronowicz, S.L.: Differential calculus on compact matrix pseudogroups (quantum groups). Commun Math. Phys. 122, 125-170 (1989) 
